\title{
A Strategy for Hedging Risks Associated with Period and Cohort Effects Using q-Forwards
}

\author{
Yanxin Liu and Johnny Siu-Hang Li
}

\begin{abstract}
The stochastic nature of future mortality arises from both period (time-related) and cohort (year-of-birth-related) effects. Existing index-based longevity hedging strategies mitigate the risk associated with period effects, but often overlook cohort effects. The negligence of cohort effects may lead to sub-optimal hedge effectiveness, if the liability being hedged is a deferred pension or annuity which involves cohorts that are not covered by the data sample. In this paper, we propose a new hedging strategy that incorporates both period and cohort effects. The resulting longevity hedge is a value hedge, reducing the uncertainty surrounding the $\tau$ year ahead value of the liability being hedged. The proposed method is illustrated with data from the male population of England and Wales. It is found that the benefit of incorporating cohort effects into a longevity hedging strategy depends heavily on the persistence of cohort effects and the choice of q-forwards.
\end{abstract}

Key words: Index-based longevity hedges; Longevity risk; Model M7; q-forwards

\section{Introduction}

Solutions for hedging pure longevity risk can be broadly divided into two categories: customized and index-based. A customized longevity hedge is based on the actual mortality experience of the individuals associated with the liability being hedged, whereas an index-based longevity hedge is linked to a broad-based mortality index which reflects the actual mortality experience of a larger pool of individuals, such as a national population. Each of these two types of longevity hedge has its pros and cons.

A customized hedge eliminates all longevity risk, but it is more costly and difficult to unwind. It is also less attractive to capital markets investors, because it lacks liquidity and transparency. Typically, a customized hedge is structured as a 'cash flow hedge', in which net payments are made period by period to immunize the longevity risk associated with every single cash flow of the hedger. An example of a customized cash flow hedge is the longevity swap that was executed between J.P. Morgan and Canada Life in 2009. Under this 40-year longevity swap, Canada Life receives from J.P. Morgan the actual payments it must pay to its annuitants and, in return, makes a series of fixed payments to J.P. Morgan (Blake et al., 2013). 
In contrast, an index-based hedge is not perfect, leaving behind residual risks such as small sample risk and population basis risk. The problems arising from these residual risks have been analyzed by researchers including Cairns et al. (2014), Coughlan et al. (2011), Haberman et al. (2014), Li and Hardy (2011) and Ngai and Sherris (2011). Nevertheless, an index-based hedge is potentially more liquid and consequently cheaper due to a lower illiquidity premium. If the hedge is adjusted periodically, then the hedging instruments used can be shorter-dated, better meeting investors' preference and reducing the hedger's exposure to counterparty default risk at the expense of increased rollover risk (see, e.g., Cairns, 2011; Zhou and Li, 2014). Furthermore, because the value of a pension or annuity liability depends on the level of the relevant mortality index at the time of valuation, index-based hedges can be structured as a 'value hedge', which immunizes the risk associated with the liability's value rather than the liability's cash flows. An example of an index-based value hedge is the q-forward contract that Lucida executed with J.P. Morgan to lock in the value of its annuity liability at the end of the hedging horizon (Blake et al., 2013).

As Coughlan et al. (2013) pointed out, index-based value hedges are very well suited for de-risking deferred liabilities, which involve no cash flow during the period of deferral. They permit pension plan sponsors with a large number of non-pensioner members (who are still years from receiving their pension benefits) to offload their longevity risk exposures. As an index-based value hedge is quite flexible, it can be adjusted from time to time according to, for example, the changes in the composition of plan sponsor's workforce and the options (e.g., a lump sum vs. lifetime payments) chosen by the plan members. Index-based value hedges also provide a way for insurers selling advanced-life delayed annuities (i.e., deeply deferred life annuities) to reduce their longevity risk exposures and hence the associated solvency capital. ${ }^{1}$ The world's first index-based value hedge for deferred members of a pension plan was the q-forward deal executed between the Pall (UK) Pension Fund and J.P. Morgan in 2011 (Blake et al., 2014). The hedge was calibrated to lock in the value of the deferred pension liability over a 10-year horizon.

Although the suitability for deferred pension and annuity liabilities is an important feature of index-based value hedges, this feature has not been extensively studied in the literature. From a technical viewpoint, deferred liabilities often involve cohorts that are not covered by the data sample to which the underlying mortality model is calibrated. To illustrate, let us suppose that the underlying mortality model is calibrated to data over ages 50 to 89 (a typical age range to which mortality models for pension valuation are fitted) and a sample period that includes the current year. The data sample does not cover the cohorts who are now age 49 or younger, which means when valuing the deferred liabilities for these cohorts, the cohort effects in the model must be projected. In other words, the deferred liabilities are subject to the stochastic uncertainty surrounding not only period (time-related) effects but also cohort (year-of-birth-related) effects.

\footnotetext{
${ }^{1}$ Advanced-life delayed annuities (ALDAs) are also known as longevity insurance, deferred income annuities (DIAs) and qualified longevity annuity contracts (QLACs). In the US, ALDAs are available from insurers including Guardian, New York Life, Mass Mutual, MetLife and Pacific Life. We refer interested readers to Gong and Webb (2010), Horneff et al. (2010) and Milevsky (2005) for further information concerning ALDAs.
} 
However, cohort effects are not taken into account in most of the existing methods for calibrating index-based hedges, including those proposed by Cairns (2011), Cairns et al. (2006b), Dahl (2004), Dahl and Møller (2006), Dahl et al. (2008), Liu and Li (2014), Zhou and Li (2014) and Luciano et al. (2012).

So far as we aware, Li and Luo (2011), Cairns et al. (2014) and Cairns (2013) have considered cohort effects in the context of hedging. Li and Luo (2011) incorporated the potential dependence between different cohorts into a static longevity hedge, but their set-up is a cash flow hedge that is not particularly suitable for a deferred liability. Cairns et al. (2014) and Cairns (2013) studied a value hedge for a deferred annuity that is payable to a single cohort of individuals; however, in their set-up, the cohort in question is covered by the data sample used in calibrating the simulation model, which means that the annuity liability is not subject to any cohort effect uncertainty. Therefore, their results do not indicate how cohort effect uncertainty may be mitigated if it is involved in liability being hedged. Also, the way they derive their hedging strategies depends heavily on simulations, and is therefore computationally intensive especially when multiple hedging instruments are included in the hedge portfolio.

This paper complements the literature by contributing a method for hedging the uncertainty surrounding both period and cohort effects. Using the proposed method, one can create a value hedge for a deferred annuity liability which involves cohort effects that are not yet realized as of the time when the hedge is established. The hedging instruments used are q-forwards, which may be linked to cohorts that are different from those that are associated with the annuity liability. At the user's discretion, the hedge can be executed as a static hedge (which remains unchanged over the hedging horizon) or a dynamic hedge (which is adjusted periodically over the hedging horizon). The proposed method is developed from the stochastic properties of the innovations in the assumed processes for the underlying period and cohort effects. It yields hedge ratios that can be expressed analytically in terms of (i) the variances and covariances of the innovations and (ii) the partial derivatives of the values of the hedge portfolio and the annuity liability with respect to the relevant innovations. As no simulation is required in the calculating the hedge ratios, the execution of our proposed hedging method requires minimal computational effort.

Unlike the hedging strategies proposed by Cairns (2011), Cairns et al. (2006b), Li and Luo (2011) and Zhou and Li (2014), the hedge ratios under our method are not obtained simply by matching the sensitivities of the liability and the hedge portfolio to changes in the underlying mortality. Instead, our method has a closer resemblance to the generalized state-space hedging method proposed by Liu and Li (2014), under which the optimal hedge ratios are calculated on the basis of a specific risk measure. ${ }^{2}$ Compared to the sensitivity matching approaches, this approach

\footnotetext{
${ }^{2}$ The generalized state-space hedging method requires the user to formulate the underlying stochastic mortality model in a state-space representation, comprised of an observation equation that relates the observed mortality to the latent state factors and a first order state process that governs the evolution of the latent state factors over time. It is not straightforward to express a stochastic mortality model in a state-space representation if it contains a cohort effect term. Therefore, the generalized state-space hedging method cannot be applied readily to the problem considered in this paper.
} 
to deriving hedge ratios is more suitable for hedgers who have a definite hedging objective. The risk measure we consider is variance, which is commonly used for the purpose of evaluating the effectiveness of longevity hedges (see Cairns, 2011, 2013; Cairns et al., 2014; Coughlan et al., 2011; $\mathrm{Li}$ and Hardy, 2011). We illustrate the proposed hedging method using real mortality data from a national population. The empirical work demonstrates the benefit of factoring cohort effects into a longevity hedge under different circumstances.

The remainder of this paper is organized as follows. Section 2 presents the stochastic mortality model that is assumed throughout the paper. Section 3 describes the liability being hedged and the hedging instruments. Section 4 introduces the proposed hedging method and details how the hedge ratios under the proposed method can be calculated. Section 5 defines the metrics for evaluating hedge effectiveness and explains how they can be calculated. Section 6 presents the baseline empirical results and performs several sensitivity tests. Finally, Section 7 concludes the paper with some suggestions for future research.

\section{The Assumed Model}

\section{$2.1 \quad$ Specification}

The model we use throughout the paper is Model M7, a generalized version of the original CairnsBlake-Dowd model (Cairns et al., 2006a). It has been shown to perform satisfactorily when applied to several national populations including the United States and England and Wales (Cairns et al., 2009, 2011; Dowd et al., 2010a,b).

Let $q_{x, t}$ be the probability that an individual dies between time $t-1$ and $t$ (i.e., during year $t$ ), given that he/she has survived to age $x$ at time $t-1$. Model M7 captures $q_{x, t}$ as follows:

$$
\ln \left(\frac{q_{x, t}}{1-q_{x, t}}\right)=\kappa_{t}^{(1)}+\kappa_{t}^{(2)}(x-\bar{x})+\kappa_{t}^{(3)}\left((x-\bar{x})^{2}-\hat{\sigma}_{x}^{2}\right)+\gamma_{t-x}^{(4)}
$$

where $\bar{x}$ is the average of the age range $\left[x_{a}, x_{b}\right]$ to which the model is calibrated and $\hat{\sigma}_{x}^{2}$ is the mean of $(x-\bar{x})^{2}$ over the same age range. In the model, $\kappa_{t}^{(1)}, \kappa_{t}^{(2)}$, and $\kappa_{t}^{(3)}$ are the period (time-related) effects, governing the random evolution of $q_{x, t}$ over time $t$. On the other hand, $\gamma_{t-x}^{(4)}$ is the cohort (year-of-birth) effect, which determines the random evolution of $q_{x, t}$ over the year of birth $(t-x)$ dimension. We estimate equation (2.1) by the method of binomial maximum likelihood with the following identifiability constraints to stipulate parameter uniqueness:

$$
\sum_{x, t} \gamma_{t-x}^{(4)}=0, \quad \sum_{x, t}(t-x) \gamma_{t-x}^{(4)}=0, \quad \text { and } \quad \sum_{x, t}(t-x)^{2} \gamma_{t-x}^{(4)}=0
$$

where the summations are taken over the entire age range $\left[x_{a}, x_{b}\right]$ and sample period $\left[t_{a}, t_{b}\right]$ to which the model is calibrated. The estimation procedure is performed with the StMoMo package 
in R (Villegas et al., 2016).

Following the ideas of Hunt and Blake (2015, 2016), we use the following stochastic processes to model the dynamics of the period and cohort effects:

$$
\left\{\begin{aligned}
\kappa_{t}^{(1)} & =\kappa_{t-1}^{(1)}+\mu_{0}^{(1)}+\mu_{1}^{(1)} t+\eta_{t}^{(1)} \\
\kappa_{t}^{(2)} & =\kappa_{t-1}^{(2)}+\mu_{0}^{(2)}+\eta_{t}^{(2)} \\
\kappa_{t}^{(3)} & =\kappa_{t-1}^{(3)}+\eta_{t}^{(3)}
\end{aligned}\right.
$$

and

$$
\begin{aligned}
& \gamma_{t-x}^{(4)}-\mu_{0}^{(4)}-\mu_{1}^{(4)}(t-x)-\mu_{2}^{(4)}(t-x)^{2} \\
= & \sum_{i=1}^{R} \phi_{i}^{(4)}\left(\gamma_{t-x-1}^{(4)}-\mu_{0}^{(4)}-\mu_{1}^{(4)}(t-x-i)-\mu_{2}^{(4)}(t-x-i)^{2}\right)+\eta_{t-x}^{(4)}+\sum_{i=1}^{M} \theta_{i}^{(4)} \eta_{t-x-i}^{(4)},
\end{aligned}
$$

where $\mu_{0}^{(1)}, \mu_{1}^{(1)}, \mu_{0}^{(2)}, \mu_{0}^{(4)}, \mu_{1}^{(4)}$ and $\mu_{2}^{(4)}$ are the parameters governing the drifts and deterministic trends in the period and cohort effects, $\phi_{1}^{(4)}, \ldots, \phi_{R}^{(4)}$ and $\theta_{1}^{(4)}, \ldots, \theta_{M}^{(4)}$ are respectively the AR and MA coefficients in the cohort effect process, and $\eta_{t}^{(1)}, \eta_{t}^{(2)}, \eta_{t}^{(3)}$ and $\eta_{t}^{(4)}$ are the random innovations which are assumed to be serially uncorrelated. It is further assumed that $\left(\eta_{t}^{(1)}, \eta_{t}^{(2)}, \eta_{t}^{(3)}\right)^{\prime}$ follows a trivariate normal distribution with a zero mean vector and a constant (positive definite) covariance matrix $Q, \eta_{t-x}^{(4)}$ follows a normal distribution with a zero mean and a constant variance of $\left(\sigma^{(4)}\right)^{2}$, and there is no correlation between $\left(\eta_{t}^{(1)}, \eta_{t}^{(2)}, \eta_{t}^{(3)}\right)^{\prime}$ and $\eta_{t-x}^{(4)}$. The use of these processes ensures that the resulting mortality projections are invariant with the (arbitrary) identifiability constraints used in estimating equation (2.1).

\subsection{Estimation}

The empirical work in this paper is based on the historical data from the male population of England and Wales (EW). The data are obtained from the Human Mortality Database (2015). We estimate equation (2.1) to the death and exposure counts for EW males over the age range of $\left[x_{a}, x_{b}\right]=[50,89]$ and the sample period of $\left[t_{a}, t_{b}\right]=[1941,2011] .^{3}$

The maximum likelihood estimates of $\kappa_{t}^{(1)}, \kappa_{t}^{(2)}, \kappa_{t}^{(3)}$ and $\gamma_{t-x}^{(4)}$ are displayed in Figure 1. The downward trend in $\kappa_{t}^{(1)}$ reflects a steady reduction in mortality at all ages over the sample period. The patterns of $\kappa_{t}^{(2)}$ and $\kappa_{t}^{(3)}$ respectively indicate how the gradient and curvature of the relationship between $\ln \left(q_{x, t} /\left(1-q_{x, t}\right)\right)$ and $x$ have changed since 1941 . The spike of $\gamma_{t-x}^{(4)}$ at $t-x=1918$ could attributed to the Spanish flu epidemic, which might have affected people who

\footnotetext{
${ }^{3}$ We use the age range of $[50,89]$ for several reasons. First, mortality improvement dynamics over different age ranges are very different; given the purpose of the model is to value pension liabilities, it is more appropriate not to include younger ages. Second, the age functions, $x-\bar{x}$ and $(x-\bar{x})^{2}-\hat{\sigma}_{x}^{2}$, in Model M7 do not capture the 'accident hump' at younger ages. Third, the data beyond age 90 are subject to reliability issues. In particular, the population counts for EW males beyond age 89 are extrapolated rather than raw values. As a fact, the age range of $[50,89]$ is used in other studies including the work of Cairns et al. (2009).
} 

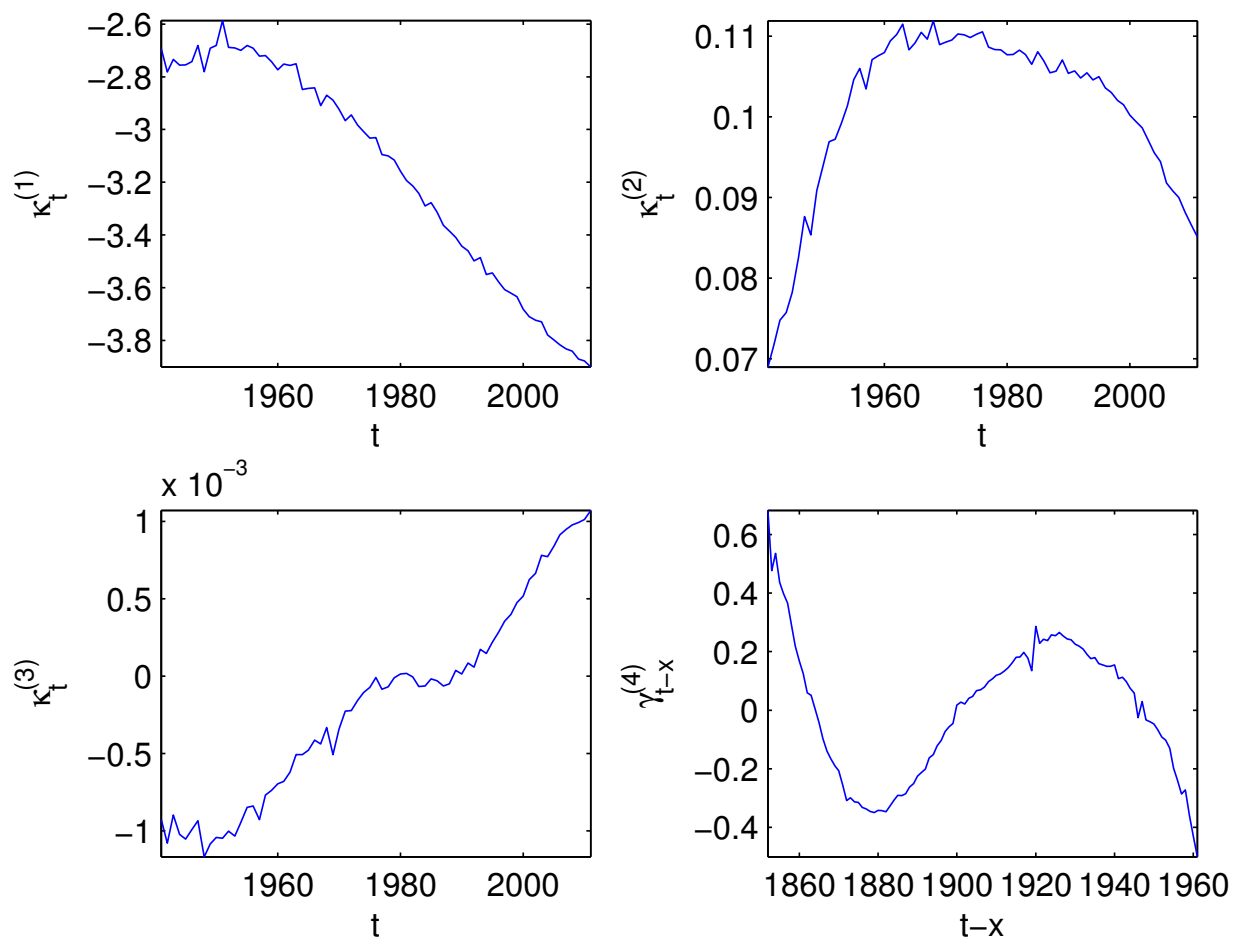

Figure 1: The maximum likelihood estimates of $\kappa_{t}^{(1)}, \kappa_{t}^{(2)}, \kappa_{t}^{(3)}$ and $\gamma_{t-x}^{(4)}$ in Model M7, $t=1941, \ldots, 2011$ and $x=50, \ldots, 89$.

were born in 1918 (see, e.g., Holmes, 2004). ${ }^{4}$

We have the estimated (realized) values of $\kappa_{t}^{(1)}, \kappa_{t}^{(2)}$ and $\kappa_{t}^{(3)}$ for $t=t_{a}=1941$ to $t=t_{b}=2011$, but the values of $\kappa_{t}^{(1)}, \kappa_{t}^{(2)}$ and $\kappa_{t}^{(3)}$ for $t>t_{b}$ are random, following the collection of processes specified in equation (2.2). For $t>t_{b}$, all values of $q_{x, t}$ are subject to the uncertainty surrounding the associated unrealized period effects. However, not all values of $q_{x, t}$ beyond the end point $t_{b}$ of the sample period are subject to cohort effect uncertainty. In particular, cohort effect uncertainty only matters to those with $t-x>t_{b}-x_{a}=2011-50=1961$, because the data sample covers years of birth up to $t_{b}-x_{a}=1961$ and consequently the cohort effects for $t-x \leq 1961$ are already realized. ${ }^{5}$ It follows that cohort effect uncertainty is irrelevant to liabilities that are payable to individuals born in or before 1961, but plays a role in deferred pension liabilities for workforces containing individuals born after 1961.

Table 1 reports the estimates of the parameters in the processes for the period and cohort effects. Parameters $Q, \theta_{1}^{(4)}, \phi_{1}^{(4)}$ and $\sigma^{(4)}$ are involved in the hedging strategy presented in Section 4. Also, the empirical fact that $Q(1,1)$ and $\left(\sigma^{(4)}\right)^{2}$ are significantly larger than $Q(2,2)$ and $Q(2,3)$

\footnotetext{
${ }^{4}$ Recently, it has also been suggested that this anomaly is attributable to the pattern of births following the demobilisation of troops after the First World War biasing the exposure to risk in the England and Wales data (see, e.g., Richards, 2008; Cairns et al., 2016).

${ }^{5}$ For instance, $q_{50,2021}$ is subject to the uncertainty surrounding $\gamma_{1971}^{(4)}$, but $q_{80,2021}$ is free of cohort effect uncertainty as $\gamma_{1941}^{(4)}$ is already known from the data sample.
} 


\begin{tabular}{l|cc}
\hline \multicolumn{3}{|c}{ The process for $\kappa_{t}^{(1)}, \kappa_{t}^{(2)}$ and $\kappa_{t}^{(3)}$} \\
\hline$\mu_{0}^{(1)}$ & \multicolumn{3}{|c}{0.6983} \\
\hline$\mu_{1}^{(1)}$ & \multicolumn{3}{|c}{$-3.6202 \times 10^{-4}$} \\
\hline$\mu_{0}^{(2)}$ & \multicolumn{3}{|c}{$2.3089 \times 10^{-4}$} \\
\hline \multirow{2}{*}{$Q$} & $\left(\begin{array}{ccc}0.0013 & 3.5927 \times 10^{-5} & 1.2299 \times 10^{-6} \\
3.5927 \times 10^{-5} & 3.4773 \times 10^{-6} & 4.6352 \times 10^{-8} \\
1.2299 \times 10^{-6} & 4.6352 \times 10^{-8} & 5.3734 \times 10^{-9}\end{array}\right)$ \\
\hline
\end{tabular}

\begin{tabular}{l|c|c|c|c|c}
\hline \multicolumn{5}{c}{ The process for $\gamma_{t-x}^{(4)}$} \\
\hline$\mu_{0}^{(4)}$ & $-3.4913 \times 10^{-10}$ & $\mu_{1}^{(4)}$ & $3.6638 \times 10^{-13}$ & $\mu_{2}^{(4)}$ & $-9.6096 \times 10^{-17}$ \\
\hline$\phi_{1}^{(4)}$ & 0.9741 & $\theta_{1}^{(4)}$ & 0.0417 & $\left(\sigma^{(4)}\right)^{2}$ & 0.0016 \\
\hline
\end{tabular}

Table 1: The estimates of the parameters in the processes for $\kappa_{t}^{(1)}, \kappa_{t}^{(2)}, \kappa_{t}^{(3)}$ and $\gamma_{t-x}^{(4)}$. The ARMA order $(R=1, M=1)$ of the cohort effect process is identified using the Box-Jenkins method (Box and Jenkins, 1976).

is used to explain some interesting properties of the hedging results in Section $6 .{ }^{6}$

\subsection{Significance of Cohort Effects}

To understand the importance of considering cohort effects, we also estimate the original CairnsBlake-Dowd model (Model M5), which does not take cohort effects into account. For each estimated model, we calculate the standardized residuals:

$$
Z_{x, t}=\frac{D_{x, t}-\hat{D}_{x, t}}{\sqrt{\operatorname{Var}\left(D_{x, t}\right)}}
$$

for $x=x_{a}, \ldots, x_{b}$ and $t=t_{a}, \ldots, t_{b}$, where $D_{x, t}$ is the actual death count at age $x$ and in year $t$ and $\hat{D}_{x, t}$ is the corresponding death count estimated from the model. ${ }^{7}$ If a model is adequate, then the pattern of $Z_{x, t}$ from the model should be random and have little clustering. In Figure 2 we compare the heat maps of $Z_{x, t}$ from Models M5 and M7. The pattern of $Z_{x, t}$ from Model M7 looks reasonably random, but that from Model M5 contains some obvious diagonal bands, indicating a lack of fit which arises from features that are year-of-birth specific. ${ }^{8}$

\footnotetext{
${ }^{6}$ We use $Q(i, j)$ to denote the $(i, j)$ th element of $Q$.

${ }^{7}$ Under the binomial death count assumption, $\operatorname{Var}\left(D_{x, t}\right)$ is given by $l_{x, t} q_{x, t}\left(1-q_{x, t}\right)$, where $l_{x, t}$ is the number of persons aged $x$ at the beginning of year $t$.

${ }^{8}$ Admittedly, the variance of $Z_{x, t}$ from Model M7 is still much larger than one (2.2191), so some large magnitude residuals (e.g., $+4,-6$ ) are observed in the diagram. One very possible reason for this problem is over-dispersion: under the binomial death count assumption, $\operatorname{Var}\left(D_{x, t}\right)=l_{x, t} q_{x, t}\left(1-q_{x, t}\right)$, which must be strictly smaller than $\mathrm{E}\left(D_{x, t}\right)=l_{x, t} q_{x, t}$. To overcome this problem, we may use instead a negative binomial death count assumption, which permits $\operatorname{Var}\left(D_{x, t}\right)$ to be greater than $\mathrm{E}\left(D_{x, t}\right)$ (Li et al., 2009). However, this alternative distributional assumption entails additional parameters (the dispersion parameters), making the model less parsimonious.
} 

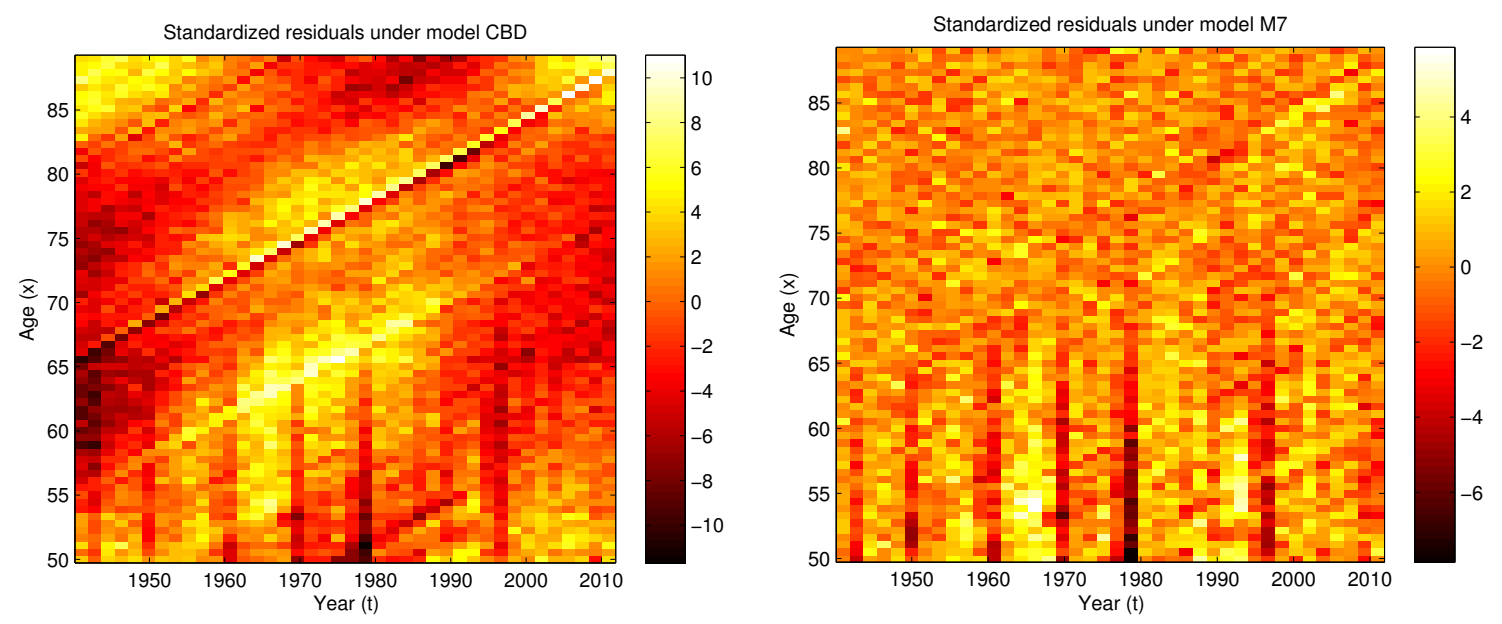

Figure 2: Heat maps of the standardized residuals $Z_{x, t}$ calculated from Models M5 and M7.

Figure 2 is reminiscent of the results produced by the Continuous Mortality Investigation Bureau (2002). They point to the conclusion that in the United Kingdom, where most of the longevity risk transfers in the world take place, cohort effects are significant and should be modeled explicitly. In the following sections, we explain how cohort effect uncertainty can be mitigated by an index-based longevity hedge, and discuss the importance of cohort effects from the perspective of hedge effectiveness.

\section{The Set-up}

\subsection{The Liability Being Hedged}

The liability being hedged is a portfolio of $T$-year deferred life annuity immediate contracts, which are sold to individuals age $x_{0}-T$ at time $t_{b}$ (the end point of the data sample period). Each contract makes no payment during the first $T$ years from time $t_{b}$; however, when the annuitant reaches age $x_{0}$ at time $t_{b}+T$, the contract pays $£ 1$ at the end of each year (starting in year $\left.t_{b}+T+1\right)$ as long as the annuitant is alive. We let $L$ be the sum of the discounted cash flows, measured at time $t_{b}$, per contract. Ignoring sampling risk, we have

$$
L=\sum_{u=1}^{\omega-x_{0}} e^{-r(T+u)} \prod_{s=1}^{T+u}\left(1-q_{x_{0}-T+s-1, t_{b}+s}\right)
$$

where $r$ is the risk-adjusted discount rate and $\omega$ is the highest attainable age. It can be seen that $L$ contains the underlying death probabilities $q_{x_{0}-T+s-1, t_{b}+s}$, for $s=1, \ldots, T+\omega-x_{0}$. Under our model assumption, this in turn means that $L$ is a function of a sequence of period effects, $\left\{\kappa_{t_{b}+s}^{(i)} ; i=1,2,3, s=1, \ldots, T+\omega-x_{0}\right\}$, and a cohort effect $\gamma_{t_{b}-\left(x_{0}-T\right)+1}^{(4)}$. 
We let $\mathcal{F}_{t}$ be the information up to and including time $t$, and define a random variable $L_{t}$ which represents $L$ given $\mathcal{F}_{t}$. The period and/or cohort effect uncertainty affecting $L_{t}$ depends on $t$ and the parameters of the annuity liability.

For $t_{b} \leq t<t_{b}+T+\omega-x_{0}, L_{t}$ is subject to the randomness of the following period effects: $\kappa_{t+1}^{(i)}, \ldots, \kappa_{t_{b}+T+\omega-x_{0}}^{(i)}$, where $i=1,2,3$. For $t>t_{b}+T+\omega-x_{0}$ (i.e., when all annuity payments have already been made), all period effects contained in $L$ are realized and therefore $L_{t}$ is no longer subject to any period effect uncertainty.

Recall that $x_{a}$ is the lower limit of the sample age range. At $t \geq t_{b}$, we have the realizations of the cohort effects up to and including year-of-birth $t-x_{a}$. As such, whether $L_{t}$ is subject to cohort effect uncertainty depends on the value of $t_{b}-\left(x_{0}-T\right)+1$ relative to that of $t-x_{a}$. If $t_{b}-\left(x_{0}-T\right)+1>t-x_{a}$, then $L_{t}$ is subject to the uncertainty associated with the unrealized value of $\gamma_{t_{b}-\left(x_{0}-T\right)+1}^{(4)}$. If $t_{b}-\left(x_{0}-T\right)+1 \leq t-x_{a}$, then $\gamma_{t_{b}-\left(x_{0}-T\right)+1}^{(4)}$ is already realized and thus $L_{t}$ is free from any cohort effect uncertainty. These relationships are illustrated in Figure 3.

Suppose that the hedge is built at time $t$, where $t \geq t_{b}$. The longevity hedge we develop is a value hedge, mitigating the uncertainty associated with

$$
V_{L_{t}}(t+\tau):=\mathrm{E}\left(L_{t} \mid \mathcal{F}_{t+\tau}\right)
$$

the value of the liability $\tau>0$ years from the time when the hedge is established. ${ }^{9}$ We assume $\tau<T+\omega-x_{0}$, as it is not reasonable to have a hedging horizon that ends after all liability cash flows are paid.

According to its definition, $V_{L_{t}}(t+\tau)$ is subject to the randomness beyond time $t$ but only up to and including time $t+\tau$. In particular, as of time $t, V_{L_{t}}(t+\tau)$ is subject to the uncertainty surrounding the random sample path of the period effects from time $t+1$ to $t+\tau$, that is, $\kappa_{t+1}^{(i)}, \ldots, \kappa_{t+\tau}^{(i)}$ for $i=1,2,3$. Also, when $t_{b}-\left(x_{0}-T\right)+1>t-x_{a}$, it is subject to the uncertainty surrounding at least part of the sample path of the cohort effects over years of birth $t+1-x_{a}$ to $t+\tau-x_{a}$, that is, $\gamma_{t+1-x_{a}}^{(4)}, \ldots, \gamma_{t+\tau-x_{a}}^{(4)} \cdot{ }^{10}$

\subsection{The Hedging Instruments}

The longevity hedge we develop consists of $m \geq 1$ q-forwards. Suppose that the q-forwards in the portfolio are launched at time $t$, where $t \geq t_{b}$. For the $j$ th q-forward, the discounted net cash

\footnotetext{
${ }^{9}$ In this paper, we perform valuation under the real-world probability measure. Therefore, the expectation in equation (3.2) is taken under the real-world probability measure and the discount rate $r$ used in (3.1) a risk-adjusted rate (rather than a risk-free rate).

${ }^{10}$ If $t_{b}-\left(x_{0}-T\right)+1>t+\tau-x_{a}$, then the expectation in $V_{L_{t}}(t+\tau)$ depends on the cohort effects up to $\gamma_{t+\tau-x_{a}}^{(4)}$ through the $\operatorname{ARMA}(R, M)$ process specified in equation (2.3). If $t_{b}-\left(x_{0}-T\right)+1 \leq t+\tau-x_{a}$, then the portion of the sample path beyond year-of-birth $t_{b}-\left(x_{0}-T\right)+1$ is irrelevant.
} 


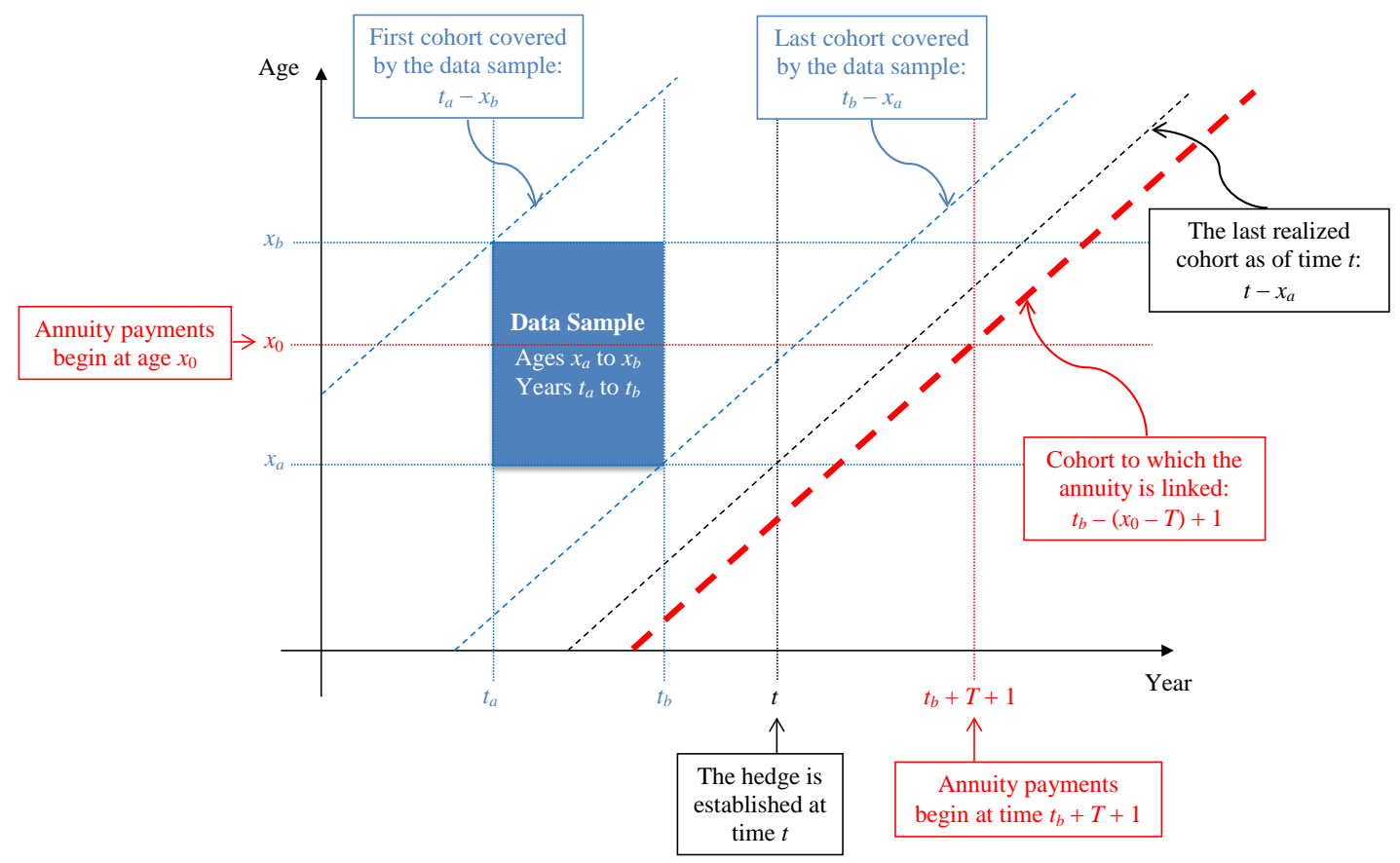

(a) $t_{b}-\left(x_{0}-T\right)+1>t-x_{a}: L_{t}$ is subject to cohort effect uncertainty

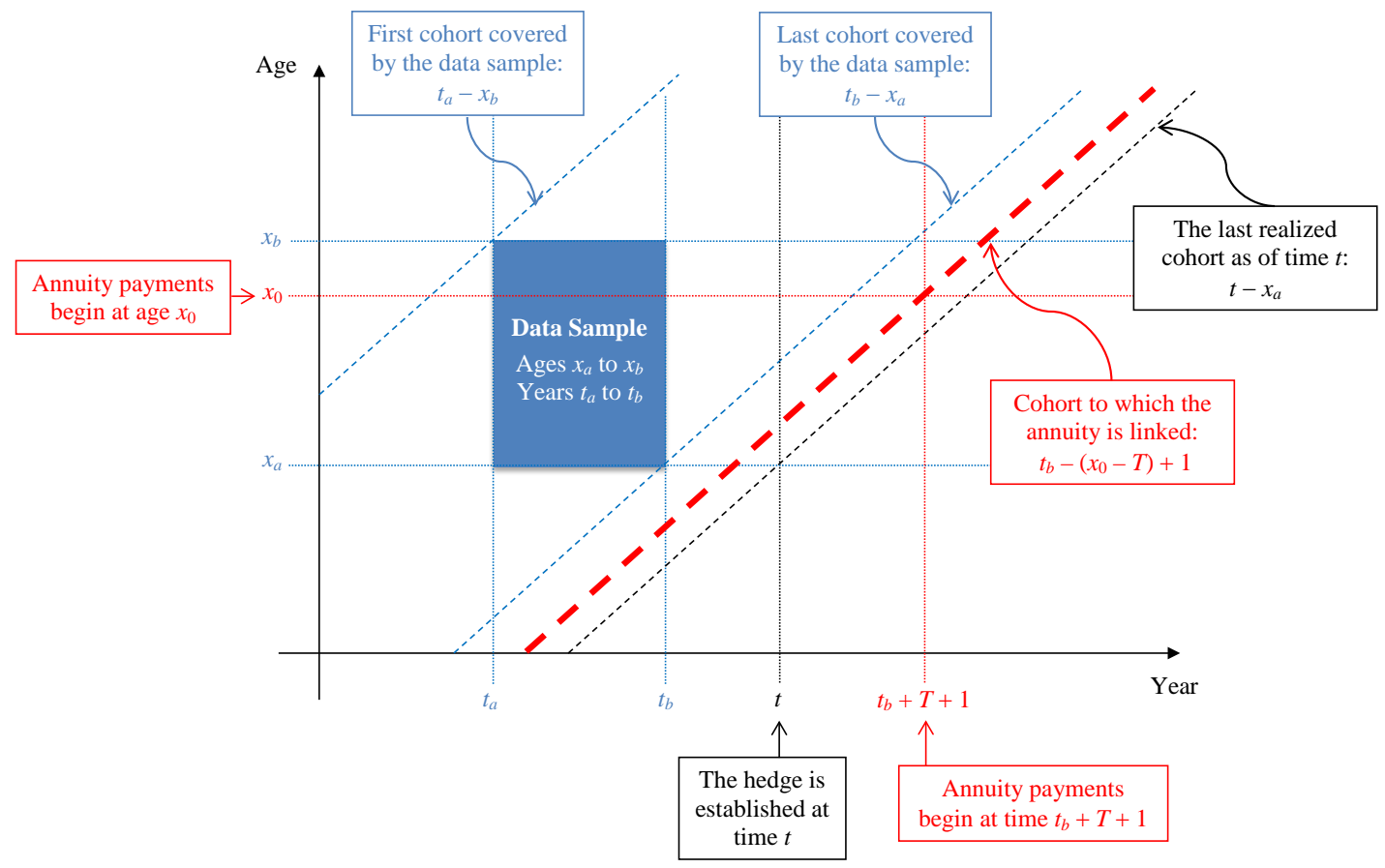

(b) $t_{b}-\left(x_{0}-T\right)+1 \leq t-x_{a}: L_{t}$ is free of cohort effect uncertainty

Figure 3: Lexis diagrams demonstrating the fact that $L_{t}$ is subject to cohort effect uncertainty if $t_{b}-\left(x_{0}-T\right)+1>t-x_{a}$ but not if $t_{b}-\left(x_{0}-T\right)+1 \leq t-x_{a}$. 
flow, measured at time $t$ and from the perspective of the fixed-rate receiver, is

$$
H(j, t)=e^{-r T_{j}}\left(q_{x_{j}, t+T_{j}}^{f}-q_{x_{j}, t+T_{j}}\right)
$$

per $£ 1$ notional, where $x_{j}, T_{j}$ and $q_{x_{j}, t+T_{j}}^{f}$ denote the reference age, the time-to-maturity and the (fixed) forward mortality rate, respectively. Under our model assumption, $H(j, t)$ is a function of $\kappa_{t+T_{j}}^{(i)}$, for $i=1,2,3$, and $\gamma_{t+T_{j}-x_{j}}^{(4)}$.

The forward mortality rate $q_{x_{j}, t+T_{j}}^{f}$ is determined at time $t$ when the q-forward is launched. We set $q_{x_{j}, t+T_{j}}^{f}$ to the best estimate of $q_{x_{j}, t+T_{j}}$, which is calculated by switching off all random innovations beyond time $t .{ }^{11}$ To focus on our research objectives, we disregard population basis risk; that is, we assume that the reference population to which the q-forwards are linked is identical to the hedger's own population of individuals. However, with moderate adaptations, the proposed hedging strategy can be extended to incorporate the difference between the populations involved.

Let $H_{t}(j, t)$ be a random variable representing $H(j, t)$ given $\mathcal{F}_{t}$. It is obvious that $H_{t}(j, t)$ is subject to the uncertainty surrounding the following (unrealized) period effects: $\kappa_{t+T_{j}}^{(i)}$, where $i=1,2,3$. Whether $H_{t}(j, t)$ is subject to cohort effect uncertainty depends on the q-forward's reference age $x_{j}$ and time-to-maturity $T_{j}$. If $t+T_{j}-x_{j}>t-x_{a}$, then $\gamma_{t+T_{j}-x_{j}}^{(4)}$ is not yet realized and hence $H_{t}(j, t)$ is subject to the uncertainty surrounding $\gamma_{t+T_{j}-x_{j}}^{(4)}$. Otherwise, $\gamma_{t+T_{j}-x_{j}}^{(4)}$ is already realized and thus $H_{t}(j, t)$ is free from any cohort effect uncertainty. The condition under which $H_{t}(j, t)$ is subject to cohort effect uncertainty is illustrated in Figure 4.

Suppose that the longevity hedge is established at time $t$, where $t \geq t_{b}$, and that all q-forwards used are freshly launched at time $t$. As the longevity hedge is built to mitigate the uncertainty associated with the value of the annuity liability at time $t+\tau$, of our particular interest is

$$
V_{H_{t}(j, t)}(t+\tau)=\mathrm{E}\left(H_{t}(j, t) \mid \mathcal{F}_{t+\tau}\right)
$$

the value of the $j$ th q-forward $\tau$ years from the time when the hedge is established. Similar to $V_{L_{t}}(t+\tau), V_{H_{t}(j, t)}(t+\tau)$ is subject to the uncertainty surrounding the sample path of the period effects from years $t+1$ to $t+\min \left(\tau, T_{j}\right)$. If $t+T_{j}-x_{j}>t-x_{a}$, then $V_{H_{t}(j, t)}(t+\tau)$ is also subject to the uncertainty surrounding the sample path of the cohort effect over years-of-birth $t+1-x_{a}$ to $\min \left(t+\tau-x_{a}, t+T_{j}-x_{j}\right)$. We remark here that if $t+T_{j}-x_{j} \leq t-x_{a}$, then the $j$ th q-forward would not be able to hedge the cohort effect uncertainty involved in the annuity liability, due to reasons that are mentioned in the previous paragraph.

\footnotetext{
${ }^{11}$ The forward mortality rate may be more realistically computed using the methods proposed by Chuang and Brockett (2014) and Li et al. (2011).
} 


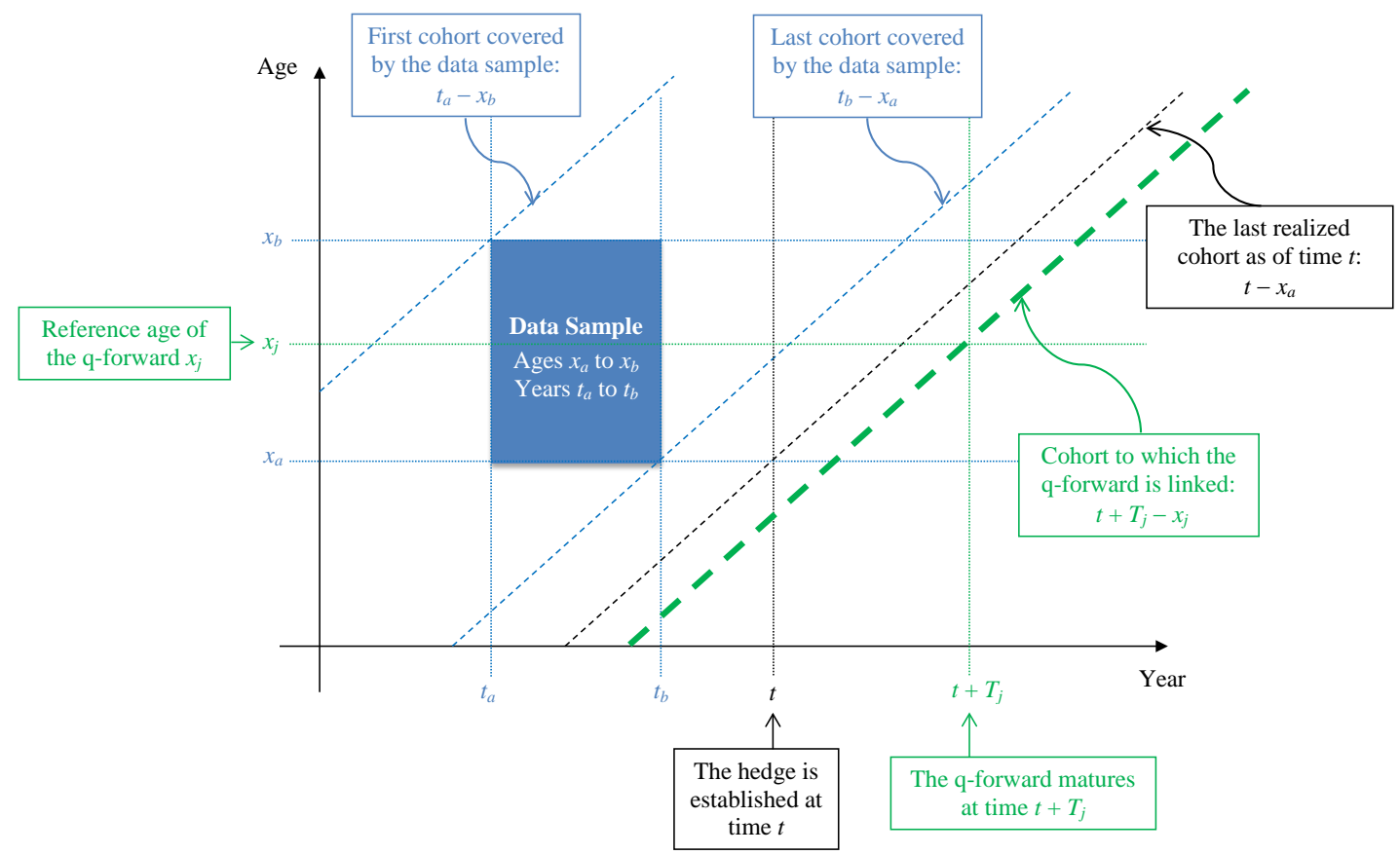

(a) $t+T_{j}-x_{j}>t-x_{a}: H_{t}(j, t)$ is subject to cohort effect uncertainty

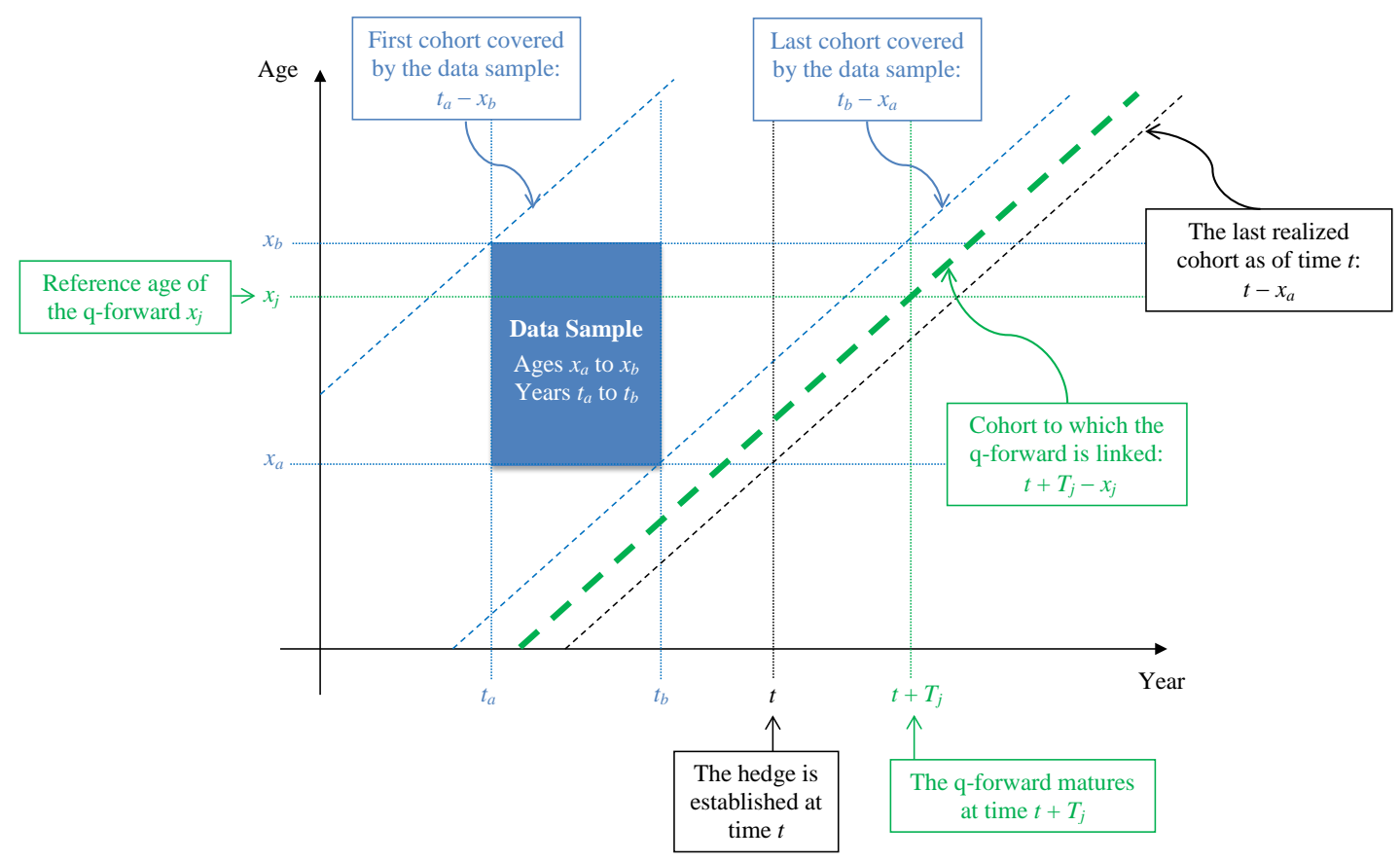

(b) $t+T_{j}-x_{j} \leq t-x_{a}: H_{t}(j, t)$ is free of cohort effect uncertainty

Figure 4: Lexis diagrams demonstrating the fact that $H_{t}(j, t)$ is subject to cohort effect uncertainty if $t+T_{j}-x_{j}>t-x_{a}$ but not if $t+T_{j}-x_{j} \leq t-x_{a}$. 


\subsection{Hedging Objectives}

\subsubsection{Static Hedging}

When we statically hedge the annuity liability, we build a q-forward portfolio at $t=t_{b}$ to mitigate the uncertainty associated with the value of the liability over a hedging horizon of $\tau>0$ years. No adjustment is made to the portfolio during the whole $\tau$-year period.

We use $N_{j}(t)$ to denote the notional amount of the $j$ th q-forward purchased at time $t$. When minimizing the variance of the $\tau$-year ahead values of the hedged position, the objective function can be expressed as

$$
\min _{N_{1}\left(t_{b}\right), \ldots, N_{m}\left(t_{b}\right)} \operatorname{Var}\left(V_{L_{t_{b}}}\left(t_{b}+\tau\right)-\sum_{j=1}^{m} N_{j}\left(t_{b}\right) V_{H_{t_{b}}\left(j, t_{b}\right)}\left(t_{b}+\tau\right) \mid \mathcal{F}_{t_{b}}\right) .
$$

\subsubsection{Dynamic Hedging}

To dynamically hedge the annuity liability, we first set up at time $t_{b}$ a q-forward portfolio that minimizes the variance of the values of the hedged position in $\tau=1$ year. At time $t_{b}+1$, the existing q-forwards are closed out and a new hedge portfolio, which is composed of q-forwards that are freshly launched at time $t_{b}+1$, is built to minimize the variance of the hedged position in the next $\tau=1$ year. The process is repeated over the desired duration, say $Y$ years.

To keep the set-up simple, we assume that the q-forwards used always have reference ages $x_{1}, \ldots x_{m}$ and times-to-maturity (measured from the launch date) $T_{1}, \ldots T_{m}$. We further assume that no transaction cost is incurred when trading the q-forward contracts. Given these assumptions, the objective function can be expressed as

$$
\min _{N_{1}(t), \ldots, N_{m}(t)} \operatorname{Var}\left(V_{L_{t}}(t+1)-\sum_{j=1}^{m} N_{j}(t) V_{H_{t}(j, t)}(t+1) \mid \mathcal{F}_{t}\right),
$$

for $t=t_{b}, t_{b}+1, \ldots, t_{b}+Y-1$.

\section{Deriving the Optimal Hedging Strategies}

\subsection{Reformulating $L_{t}$ and $H_{t}(j, t)$}

As discussed in Section 3, $L_{t}$ and $H_{t}(j, t)$ are functions of various unrealized period and/or cohort effects. However, these functional forms are not easy to work with, because there exist serial correlations in the period and cohort effects. To get around this problem, we now reformulate $L_{t}$ 
and $H_{t}(j, t)$ as functions of the innovations (in the stochastic processes for the period and cohort effects), which, by definition, are free of any serial correlation.

First, let us consider the period effects. Using equation (2.2), we can express the period effects for year $t+s$ as

$$
\left\{\begin{array}{l}
\kappa_{t+s}^{(1)}=\kappa_{t}^{(1)}+\mu_{0}^{(1)} s+\mu_{1}^{(1)} \sum_{u=1}^{s}(t+s)+\sum_{u=1}^{s} \eta_{t+u}^{(1)} \\
\kappa_{t+s}^{(2)}=\kappa_{t}^{(2)}+\mu_{0}^{(2)} s+\sum_{u=1}^{s} \eta_{t+u}^{(2)} \\
\kappa_{t+s}^{(3)}=\kappa_{t}^{(3)}+\sum_{u=1}^{s} \eta_{t+u}^{(3)}
\end{array}\right.
$$

Given $\mathcal{F}_{t}$, the only random components in the above are the following innovations: $\eta_{t+1}^{(i)}, \ldots, \eta_{t+s}^{(i)}$.

Next, we turn to the cohort effects. By rewriting equation (2.3) into an infinite MA form, we can express the cohort effect for year-of-birth $t-x_{a}+s$ as

$$
\begin{aligned}
\gamma_{t-x_{a}+s}^{(4)}= & \mu_{0}^{(4)}+\mu_{1}^{(4)}\left(t-x_{a}+s\right)+\mu_{2}^{(4)}\left(t-x_{a}+s\right)^{2} \\
& +\left(1+\sum_{i=1}^{M} \theta_{i}^{(4)} B^{i}\right)\left(1-\sum_{i=1}^{R} \phi_{i}^{(4)} B^{i}\right)^{-1} \eta_{t-x_{a}+s}^{(4)}
\end{aligned}
$$

where $B$ denotes the backshift operator. Given $\mathcal{F}_{t}$, the only random terms in the expression are the following innovations: $\eta_{t-x_{a}+1}^{(4)}, \ldots, \eta_{t-x_{a}+s}^{(4)}$.

Combining the above and the results from Section 3, we can draw the following conclusions. When $t_{b} \leq t<t_{b}+T+\omega-x_{0}$ (the range of $t$ that is of our interest), $L_{t}$ is a function of the following random innovations: $\eta_{t+1}^{(i)}, \ldots \eta_{t+T+\omega-x_{0}}^{(i)}$, where $i=1,2,3$. Also, if $t_{b}-\left(x_{0}-T\right)+1>t-x_{a}$, then $L_{t}$ is also a function of $\eta_{t-x_{a}+1}^{(4)}, \ldots, \eta_{t_{b}+T-x_{0}+1}^{(4)}$. All other components in $L_{t}$ are non-random as of time $t$.

For any $t \geq t_{b}, H_{t}(j, t)$ is a function of the following random innovations from the period effect process: $\eta_{t+1}^{(i)}, \ldots, \eta_{t+T_{j}}^{(i)}$, where $i=1,2,3$. If $t+T_{j}-x_{j}>t-x_{a}$, then $H_{t}(j, t)$ is also a function of $\eta_{t-x_{a}+1}^{(4)}, \ldots, \eta_{t+T_{j}-x_{j}}^{(4)}$. Otherwise, $H_{t}(j, t)$ involves no unrealized innovation from the cohort effect process. Other than the mentioned innovations, all components in $H_{t}(j, t)$ are non-random as of time $t$.

We conclude this sub-section by specifying the innovation vector $\vec{\eta}_{t}$ and its probability distribution. We define $\vec{\eta}_{t}$ as follows:

$$
\vec{\eta}_{t}:=\left(\eta_{t}^{(1)}, \eta_{t}^{(2)}, \eta_{t}^{(3)}, \eta_{t-x_{a}}^{(4)}\right)^{\prime}
$$

Given the model assumptions, $\vec{\eta}_{t}$ possesses no serial correlation. It follows a multivariate normal distribution with a zero mean vector and a covariance matrix

$$
Q^{*}=\left(\begin{array}{cc}
Q & 0 \\
0 & \left(\sigma^{(4)}\right)^{2}
\end{array}\right),
$$


where $Q$ and $\left(\sigma^{(4)}\right)^{2}$ are defined in Section $2 .^{12}$

\subsection{Linear Approximations}

It follows from equations (3.1) and (3.3) that both $L_{t}$ and $H_{t}(j, t)$ are non-linear functions of the period and cohort effects (and thus non-linear functions of the innovations from the period and cohort effect processes). To enable an analytical derivation of the hedging strategies, we approximate $L_{t}$ and $H_{t}(j, t)$ with first-order Taylor expansions around the relevant random innovations.

We let $l_{t}$ and $h_{t}(j, t)$ be the first-order Taylor approximations of $L_{t}$ and $H_{t}(j, t)$, respectively. We have

$$
l_{t}=\hat{L}_{t}+\sum_{i=1}^{3} \sum_{s=1}^{T+\omega-x_{0}-t} \frac{\partial L_{t}}{\partial \eta_{t+s}^{(i)}} \eta_{t+s}^{(i)}+\sum_{s=1}^{t_{b}-\left(x_{0}-T\right)+x_{a}-t+1} \frac{\partial L_{t}}{\partial \eta_{t-x_{a}+s}^{(4)}} \eta_{t-x_{a}+s}^{(4)},
$$

for $t_{b} \leq t<t_{b}+T+\omega-x_{0}$, and

$$
h_{t}(j, t)=\hat{H}_{t}(j, t)+\sum_{i=1}^{3} \sum_{s=1}^{T_{j}} \frac{\partial H_{t}(j, t)}{\partial \eta_{t+s}^{(i)}} \eta_{t+s}^{(i)}+\sum_{s=1}^{T_{j}-x_{j}} \frac{\partial H_{t}(j, t)}{\partial \eta_{t-x_{a}+s}^{(4)}} \eta_{t-x_{a}+s}^{(4)},
$$

for $t \geq t_{b}$, where $\hat{L}_{t}$ and $\hat{H}_{t}(j, t)$ respectively represent the estimates of $L_{t}$ and $H_{j, t}$ that are computed by setting $\vec{\eta}_{t+s}$ to $\overrightarrow{0}$ (the expected value of $\vec{\eta}_{t+s}$ ) for all $s>0$. Also, throughout this paper, all partial derivatives with respect to the innovations are evaluated at either the realized innovations (if the innovations are realized) or the expected values of the innovations (if the innovations are not yet realized).

Using equation (4.3), we obtain the following approximation for $V_{L_{t}}(t+\tau)$ :

$$
\begin{aligned}
V_{L_{t}}(t+\tau) & \approx V_{l_{t}}(t+\tau) \\
& :=\mathrm{E}\left(l_{t} \mid \mathcal{F}_{t+\tau}\right) \\
& =\mathrm{E}\left(\hat{L}_{t}+\sum_{i=1}^{3} \sum_{s=1}^{T+\omega-x_{0}-t} \frac{\partial L_{t}}{\partial \eta_{t+s}^{(i)}} \eta_{t+s}^{(i)}+\sum_{s=1}^{t_{b}-\left(x_{0}-T\right)+x_{a}-t+1} \frac{\partial L_{t}}{\partial \eta_{t-x_{a}+s}^{(4)}} \eta_{t-x_{a}+s}^{(4)} \mathcal{F}_{t+\tau}\right) \\
& =\hat{L}_{t}+\sum_{i=1}^{3} \sum_{s=1}^{\tau} \frac{\partial L_{t}}{\partial \eta_{t+s}^{(i)}} \eta_{t+s}^{(i)}+\sum_{s=1}^{\tau} \frac{\partial L_{t}}{\partial \eta_{t-x_{a}+s}^{(4)}} \eta_{t-x_{a}+s}^{(4)} \\
& =\hat{L}_{t}+\sum_{s=1}^{\tau}\left(\frac{\partial L_{t}}{\partial \vec{\eta}_{t+s}}\right)^{\prime} \vec{\eta}_{t+s}
\end{aligned}
$$

where $\frac{\partial L_{t}}{\partial \vec{\eta}_{t+s}}=\left(\frac{\partial L_{t}}{\partial \eta_{t+s}^{(1)}}, \frac{\partial L_{t}}{\partial \eta_{t+s}^{(2)}}, \frac{\partial L_{t}}{\partial \eta_{t+s}^{(3)}}, \frac{\partial L_{t}}{\partial \eta_{t-x_{a}+s}^{(4)}}\right)^{\prime}$

\footnotetext{
${ }^{12}$ In the original Model M7 (Cairns et al., 2009), the period and cohort effects are assumed to be uncorrelated with each other. The two block diagonal matrices in $Q^{*}$ reflect this assumption.
} 
Also, using equation (4.4), we have the following approximation for $V_{H_{t}(j, t)}(t+\tau)$ :

$$
\begin{aligned}
V_{H_{t}(j, t)}(t+\tau) & \approx V_{h_{t}(j, t)}(t+\tau) \\
& :=\mathrm{E}\left(h_{t}(j, t) \mid \mathcal{F}_{t}\right) \\
& =\mathrm{E}\left(\hat{H}_{t}(j, t)+\sum_{i=1}^{3} \sum_{s=1}^{T_{j}} \frac{\partial H_{t}(j, t)}{\partial \eta_{t+s}^{(i)}} \eta_{t+s}^{(i)}+\sum_{s=1}^{T_{j}-x_{j}} \frac{\partial H_{t}(j, t)}{\partial \eta_{t-x_{a}+s}^{(4)}} \eta_{t-x_{a}+s}^{(4)} \mid \mathcal{F}_{t+\tau}\right) \\
& =\hat{H}_{t}(j, t)+\sum_{i=1}^{3} \sum_{s=1}^{\tau} \frac{\partial H_{t}(j, t)}{\partial \eta_{t+s}^{(i)}} \eta_{t+s}^{(i)}+\sum_{s=1}^{\tau} \frac{\partial H_{t}(j, t)}{\partial \eta_{t-x_{a}+s}^{(4)}} \eta_{t-x_{a}+s}^{(4)} \\
& =\hat{H}_{t}(j, t)+\sum_{s=1}^{\tau}\left(\frac{\partial H_{t}(j, t)}{\partial \vec{\eta}_{t+s}}\right)^{\prime} \vec{\eta}_{t+s},
\end{aligned}
$$

where $\frac{\partial H_{t}(j, t)}{\partial \vec{\eta}_{t+s}}=\left(\frac{\partial H_{t}(j, t)}{\partial \eta_{t+s}^{(1)}}, \frac{\partial H_{t}(j, t)}{\partial \eta_{t+s}^{(2)}}, \frac{\partial H_{t}(j, t)}{\partial \eta_{t+s}^{(3)}}, \frac{\partial H_{t}(j, t)}{\partial \eta_{t-x_{a}+s}^{(4)}}\right)^{\prime}$

The expression for $V_{l_{t}}(t+\tau)$ contains the partial derivatives of $L_{t}$ with respect to different innovations. Those with respect to the period-effect-related innovations can be computed analytically as follows:

$$
\begin{aligned}
\frac{\partial L_{t}}{\partial \eta_{t+k}^{(1)}}= & -\sum_{u=1}^{\omega-x_{0}} e^{-r(T+u)}\left(\prod_{s=1}^{T+u}\left(1-q_{x_{0}-T+s-1, t_{b}+s}\right)\right)\left(\sum_{s=t-t_{b}+k}^{T+u} q_{x_{0}-T+s-1, t_{b}+s}\right) ; \\
\frac{\partial L_{t}}{\partial \eta_{t+k}^{(2)}}= & -\sum_{u=1}^{\omega-x_{0}} e^{-r(T+u)}\left(\prod_{s=1}^{T+u}\left(1-q_{x_{0}-T+s-1, t_{b}+s}\right)\right) \\
& \times\left(\sum_{s=t-t_{b}+k}^{T+u}\left(x_{0}-T+s-1-\bar{x}\right) q_{x_{0}-T+s-1, t_{b}+s}\right) ; \\
\frac{\partial L_{t}}{\partial \eta_{t+k}^{(3)}=} & -\sum_{u=1}^{\omega-x_{0}} e^{-r(T+u)}\left(\prod_{s=1}^{T+u}\left(1-q_{x_{0}-T+s-1, t_{b}+s}\right)\right) \\
& \times\left(\sum_{s=t-t_{b}+k}^{T+u}\left(\left(x_{0}-T+s-1-\bar{x}\right)^{2}-\hat{\sigma}_{x}^{2}\right) q_{x_{0}-T+s-1, t_{b}+s}\right),
\end{aligned}
$$

for $k=1, \ldots, \omega-x_{0}+T+\left(t_{b}-t\right)$, and

$$
\frac{\partial L_{t}}{\partial \eta_{t+k}^{(1)}}=\frac{\partial L_{t}}{\partial \eta_{t+k}^{(2)}}=\frac{\partial L_{t}}{\partial \eta_{t+k}^{(3)}}=0
$$

for $k>\omega-x_{0}+T+\left(t_{b}-t\right)$. Those with respect to the cohort-effect-related innovations can be computed analytically as follows:

$$
\frac{\partial L_{t}}{\partial \eta_{t-x_{a}+k}^{(4)}}=-X\left(C_{t, k}\right) \sum_{u=1}^{\omega-x_{0}} e^{-r(T+u)}\left(\prod_{s=1}^{T+u}\left(1-q_{x_{0}-T+s-1, t_{b}+s}\right)\right)\left(\sum_{s=t-t_{b}+1}^{T+u} q_{x_{0}-T+s-1, t_{b}+s}\right)
$$


for $k=1, \ldots, t_{b}-x_{0}+T+1-t+x_{a}$, where $C_{t, k}=t_{b}-\left(x_{0}-T\right)+1-\left(t-x_{a}+k\right)$ and

$$
X(s)= \begin{cases}0, & \text { if } s<0 \\ 1, & \text { if } s=0 \\ \phi_{1}^{(4)}+\theta_{1}^{(4)}, & \text { if } s=1 \\ \sum_{i=1}^{s-1} \phi_{i}^{(4)} X(s-i)+\left(\phi_{s}^{(4)}+\theta_{s}^{(4)}\right), & \text { if } s \geq 2\end{cases}
$$

with $\phi_{s}^{(4)}=0$ if $s>R$ and $\theta_{s}^{(4)}=0$ if $s>M$, and

$$
\frac{\partial L_{t}}{\partial \eta_{t-x_{a}+k}^{(4)}}=0
$$

for $k>t_{b}-x_{0}+T+1-t+x_{a} \cdot{ }^{13}$

The expression for $V_{h_{t}(j, t)}(t+\tau)$ contains the partial derivatives of $H_{t}(j, t)$ with respect to different innovations. Those respect to the period-effect-related innovations can be calculated analytically as follows:

$$
\begin{aligned}
\frac{\partial H_{t}(j, t)}{\partial \eta_{t+k}^{(1)}} & =-e^{-r T_{j}}\left(1-q_{x_{j}, t+T_{j}}\right) q_{x_{j}, t+T_{j}} ; \\
\frac{\partial H_{t}(j, t)}{\partial \eta_{t+k}^{(2)}} & =-e^{-r T_{j}}\left(x_{j}-\bar{x}\right)\left(1-q_{x_{j}, t+T_{j}}\right) q_{x_{j}, t+T_{j}} ; \\
\frac{\partial H_{t}(j, t)}{\partial \eta_{t+k}^{(3)}} & =-e^{-r T_{j}}\left(\left(x_{j}-\bar{x}\right)^{2}-\hat{\sigma}_{x}^{2}\right)\left(1-q_{x_{j}, t+T_{j}}\right) q_{x_{j}, t+T_{j}},
\end{aligned}
$$

for $k=1, \ldots, T_{j}$, and

$$
\frac{\partial H_{t}(j, t)}{\partial \eta_{t+k}^{(1)}}=\frac{\partial H_{t}(j, t)}{\partial \eta_{t+k}^{(2)}}=\frac{\partial H_{t}(j, t)}{\partial \eta_{t+k}^{(3)}}=0,
$$

for $k>T_{j}$. Those with respect to the cohort-effect-related innovations can be calculated analytically as follows:

$$
\frac{\partial H_{t}(j, t)}{\partial \eta_{t-x_{a}+k}^{(4)}}=-X\left(T_{j}-x_{j}+x_{a}-k\right) e^{-r T_{j}}\left(1-q_{x_{j}, t+T_{j}}\right) q_{x_{j}, t+T_{j}}
$$

for $k=1, \ldots, T_{j}-x_{j}+x_{a}$ and

$$
\frac{\partial H_{t}(j, t)}{\partial \eta_{t-x_{a}+k}^{(4)}}=0
$$

for $k>T_{j}-x_{j}+x_{a} \cdot{ }^{14}$ The derivation of the partial derivatives of $L_{t}$ and $H_{t}(j . t)$ is provided in

\footnotetext{
${ }^{13}$ As the annuity liability is associated with the cohort born in $t_{b}-x_{0}+T+1$, it is free of the cohort effect uncertainty beyond year-of-birth $t_{b}-x_{0}+T+1$. Hence, the partial derivative of $L_{t}$ with respect to $\eta_{t-x_{a}+k}^{(4)}$ is 0 when $t-x_{a}+k>t_{b}-x_{0}+T+1$ or equivalently $k>t_{b}-x_{0}+T+1-t+x_{a}$.

${ }^{14}$ As the q-forward is linked to year-of-birth $t+T_{j}-x_{j}$, it is irrelevant to any cohort effect uncertainty beyond yearof-birth $t+T_{j}-x_{j}$. Therefore, the partial derivative of $H_{t}(j, t)$ with respect to $\eta_{t-x_{a}+k}^{(4)}$ is 0 for $t-x_{a}+k>t+T_{j}-x_{j}$
} 
Appendices A and B.

\subsection{Minimizing Variance}

As discussed in Section 3.3, the variance to be minimized is

$$
\operatorname{Var}\left(V_{L_{t}}(t+\tau)-\sum_{j=1}^{m} N_{j}(t) V_{H_{t}(j, t)}(t+\tau) \mid \mathcal{F}_{t}\right),
$$

where $t=t_{b}$ for a static hedge, and $t=t_{b}, \ldots, T$ and $\tau=1$ for a dynamic hedge.

Using equations (4.5) and (4.6), the variance to be minimized can be approximated as

$$
\begin{aligned}
& \operatorname{Var}\left(V_{l_{t}}(t+\tau)-\sum_{j=1}^{m} N_{j}(t) V_{h_{t}(j, t)}(t+\tau) \mid \mathcal{F}_{t}\right) \\
= & \sum_{s=1}^{\tau}\left(\frac{\partial L_{t}}{\partial \vec{\eta}_{t+s}}-\sum_{j=1}^{m} N_{j}(t) \frac{\partial H_{t}(j, t)}{\partial \vec{\eta}_{t+s}}\right)^{\prime} Q^{*}\left(\frac{\partial L_{t}}{\partial \vec{\eta}_{t+s}}-\sum_{j=1}^{m} N_{j}(t) \frac{\partial H_{t}(j, t)}{\partial \vec{\eta}_{t+s}}\right) .
\end{aligned}
$$

We can calculate the optimal notional amounts $\hat{N}_{1}(t), \ldots, \hat{N}_{m}(t)$ by solving the first-order conditions, that is, by setting the first partial derivatives of (4.12) with respect to $N_{1}(t), \ldots, N_{m}(t)$ to zero. The solution is given by

$$
\left(\begin{array}{c}
\hat{N}_{1}(t) \\
\vdots \\
\hat{N}_{m}(t)
\end{array}\right)=\left(\begin{array}{ccc}
\sum_{s=1}^{\tau}\left(\frac{\partial H_{t}(1, t)}{\partial \vec{\eta}_{t+s}}\right)^{\prime} Q^{*} \frac{\partial H_{t}(1, t)}{\partial \vec{\eta}_{t+s}} & \cdots & \sum_{s=1}^{\tau}\left(\frac{\partial H_{t}(1, t)}{\partial \vec{\eta}_{t+s}}\right)^{\prime} Q^{*} \frac{\partial H_{t}(m, t)}{\partial \vec{\eta}_{t+s}} \\
\vdots & \ddots & \vdots \\
\sum_{s=1}^{\tau}\left(\frac{\partial H_{t}(m, t)}{\partial \vec{\eta}_{t+s}}\right)^{\prime} Q^{*} \frac{\partial H_{t}(1, t)}{\partial \vec{\eta}_{t+s}} & \cdots & \sum_{s=1}^{\tau}\left(\frac{\partial H_{t}(m, t)}{\partial \vec{\eta}_{t+s}}\right)^{\prime} Q^{*} \frac{\partial H_{t}(m, t)}{\partial \vec{\eta}_{t+s}}
\end{array}\right)^{-1}\left(\begin{array}{c}
\sum_{s=1}^{\tau}\left(\frac{\partial H_{t}(1, t)}{\partial \vec{\eta}_{t+s}}\right)^{\prime} Q^{*} \frac{\partial L_{t}}{\partial \vec{\eta}_{t+s}} \\
\vdots \\
\sum_{s=1}^{\tau}\left(\frac{\partial H_{t}(m, t)}{\partial \vec{\eta}_{t+s}}\right)^{\prime} Q^{*} \frac{\partial L_{t}}{\partial \vec{\eta}_{t+s}}
\end{array}\right)
$$

The second order conditions (not shown) indicate that the solution above minimizes the variance specified in equation (4.12).

To develop a deeper insight into the solution, let us focus on the case when $m=1$ q-forward or equivalently $k>T_{j}-x_{j}+x_{a}$. 
is used. In this case, the variance to be minimized reduces to

$$
\begin{aligned}
& \operatorname{Var}\left(V_{l_{t}}(t+\tau)-N_{1}(t) V_{h_{t}(1, t)}(t+\tau) \mid \mathcal{F}_{t}\right) \\
= & \sum_{s=1}^{\tau}\left(\frac{\partial L_{t}}{\partial \vec{\eta}_{t+s}}-N_{1}(t) \frac{\partial H_{t}(1, t)}{\partial \vec{\eta}_{t+s}}\right)^{\prime} Q^{*}\left(\frac{\partial L_{t}}{\partial \vec{\eta}_{t+s}}-N_{1}(t) \frac{\partial H_{t}(1, t)}{\partial \vec{\eta}_{t+s}}\right) \\
= & \sum_{s=1}^{\tau}\left(\frac{\partial L_{t}}{\partial \vec{\eta}_{t+s}}\right)^{\prime} Q^{*}\left(\frac{\partial L_{t}}{\partial \vec{\eta}_{t+s}}\right)-2 N_{1}(t) \sum_{s=1}^{\tau}\left(\frac{\partial H_{t}(1, t)}{\partial \vec{\eta}_{t+s}}\right)^{\prime} Q^{*}\left(\frac{\partial L_{t}}{\partial \vec{\eta}_{t+s}}\right) \\
& +\left(N_{1}(t)\right)^{2} \sum_{s=1}^{\tau}\left(\frac{\partial H_{t}(1, t)}{\partial \vec{\eta}_{t+s}}\right)^{\prime} Q^{*}\left(\frac{\partial H_{t}(1, t)}{\partial \vec{\eta}_{t+s}}\right) \\
= & k_{3}-2 N_{1}(t) \cdot k_{2}+\left(N_{1}(t)\right)^{2} \cdot k_{1} \\
= & k_{1}\left(N_{1}(t)-\frac{k_{2}}{k_{1}}\right)^{2}+k_{3}-\frac{k_{2}^{2}}{k_{1}},
\end{aligned}
$$

where

$$
k_{1}=\sum_{s=1}^{\tau}\left(\frac{\partial H_{t}(1, t)}{\partial \vec{\eta}_{t+s}}\right)^{\prime} Q^{*}\left(\frac{\partial H_{t}(1, t)}{\partial \vec{\eta}_{t+s}}\right)
$$

is the approximate variance of $H_{t}(1, t)$,

$$
k_{2}=\sum_{s=1}^{\tau}\left(\frac{\partial H_{t}(1, t)}{\partial \vec{\eta}_{t+s}}\right)^{\prime} Q^{*}\left(\frac{\partial L_{t}}{\partial \vec{\eta}_{t+s}}\right)
$$

is the approximate covariance between $H_{t}(1, t)$ and $L_{t}$, and

$$
k_{3}=\sum_{s=1}^{\tau}\left(\frac{\partial L_{t}}{\partial \vec{\eta}_{t+s}}\right)^{\prime} Q^{*}\left(\frac{\partial L_{t}}{\partial \vec{\eta}_{t+s}}\right)
$$

is the approximate variance of $L_{t}$. It becomes clear that the optimal notional amount for $m=1$ is

$$
\hat{N}_{1}(t)=\frac{k_{2}}{k_{1}},
$$

and the minimized variance is

$$
k_{3}-\frac{k_{2}^{2}}{k_{1}}
$$

How would the solution change if relevant cohort effect uncertainty is not taken into account? Suppose that cohort effect does exist but the hedger mistakenly ignores cohort effect uncertainty (i.e., treating $\left(\sigma^{(4)}\right)^{2}$ as zero). In this hypothetical situation, the expression of the optimized notional amount would exclude the terms involving $\left(\sigma^{(4)}\right)^{2}$ and become

$$
\hat{\mathcal{N}}_{1}(t)=\frac{k_{2}-c_{2}}{k_{1}-c_{1}}=\frac{k_{2}\left(1-\frac{c_{2}}{k_{2}}\right)}{k_{1}\left(1-\frac{c_{1}}{k_{1}}\right)},
$$


where

$$
c_{1}=\sum_{s=1}^{\tau}\left(\frac{\partial H_{t}(j, t)}{\partial \eta_{t-x_{a}+s}^{(4)}}\right)^{2}\left(\sigma^{(4)}\right)^{2}
$$

can be viewed as the portion of the variance of $H_{j}(j, t)$ that is contributed from the innovations associated with the cohort effects, and

$$
c_{2}=\sum_{s=1}^{\tau} \frac{\partial H_{t}(j, t)}{\partial \eta_{t-x_{a}+s}^{(4)}} \cdot \frac{\partial L_{t}}{\partial \eta_{t-x_{a}+s}^{(4)}}\left(\sigma^{(4)}\right)^{2}
$$

can be understood as the portion of the covariance between $H_{t}(j, t)$ and $L_{t}$ that is contributed from the innovations associated with the cohort effects. It is clear that $\hat{\mathcal{N}}_{1}(t)$ is either larger or smaller than the actual optimal notional amount $\hat{N}_{1}(t)$ (which incorporates cohort effect uncertainty) unless $c_{1} / k_{1}=c_{2} / k_{2}$. It can also be seen that $\hat{\mathcal{N}}_{1}(t)$ is close to $\hat{N}_{1}(t)$ when $c_{1} / k_{1}$ and $c_{2} / k_{2}$ are close to zero. The conditions under which under which $\hat{\mathcal{N}}_{1}(t)$ and $\hat{N}_{1}(t)$ are equal (or close to each other) are further discussed in Section 6 when we present the numerical results. Substituting $N_{1}(t)=\hat{\mathcal{N}}_{1}(t)$ into equation (4.14), we obtain the variance of the values of the hedged position when cohort effect uncertainty is ignored in the calibration procedure but it actually exists:

$$
k_{3}-2 k_{2} \frac{k_{2}-c_{2}}{k_{1}-c_{1}}+k_{1}\left(\frac{k_{2}-c_{2}}{k_{1}-c_{1}}\right)^{2} .
$$

The difference between expressions (4.20) and (4.19) is

$$
\frac{1}{k_{1}}\left(k_{2}-k_{1} \frac{k_{2}-c_{2}}{k_{1}-c_{1}}\right)^{2}
$$

which is strictly greater than zero unless $c_{1} / k_{1}=c_{2} / k_{2} \cdot{ }^{15}$ It follows that ignoring cohort effect uncertainty generally leads to a sub-optimal variance minimization.

\section{$5 \quad$ Evaluating Hedge Effectiveness}

We measure hedge effectiveness as the proportion of variance reduced. For a static hedge, the metric can be expressed as

$$
\mathrm{HE}^{\operatorname{Var}}=1-\frac{\operatorname{Var}\left(V_{L_{t_{b}}}\left(t_{b}+\tau\right)-\sum_{j=1}^{m} N_{j}\left(t_{b}\right) V_{H_{t_{b}}\left(j, t_{b}\right)}\left(t_{b}+\tau\right) \mid \mathcal{F}_{t_{b}}\right)}{\operatorname{Var}\left(V_{L_{t_{b}}}\left(t_{b}+\tau\right) \mid \mathcal{F}_{t_{b}}\right)} .
$$

\footnotetext{
${ }^{15}$ We can interpret $k_{1}$ as the approximate variance of $H_{j}(j, t)$, and thus it is strictly greater than zero.
} 
For a dynamic hedge, the metric (applicable to year $t+1$ ) is given by

$$
\mathrm{HE}_{t}^{\mathrm{Var}}=1-\frac{\operatorname{Var}\left(V_{L_{t}}(t+1)-\sum_{j=1}^{m} N_{j}(t) V_{H_{t}(j, t)}(t+1) \mid \mathcal{F}_{t}\right)}{\operatorname{Var}\left(V_{L_{t}}(t+1) \mid \mathcal{F}_{t}\right)},
$$

for $t=t_{b}, t_{b}+1, \ldots, t_{b}+Y-1$. The value of this metric is close to 1 if the longevity hedge is effective, and close to 0 if otherwise.

Both metrics can be computed using analytical approximations. In particular, we can use equation (4.12) to approximately calculate metrics (5.1) and (5.2). This method requires no simulation.

\section{$6 \quad$ Numerical Illustrations}

\subsection{The Baseline Results}

Let us begin by stating the baseline assumptions. In line with the set-up described in Section 3, the liabilities being hedged are deferred life annuities that are sold at the end of year $t_{b}=2011$ when the data sample period terminates. For all annuities, the age at which the period of deferral ends is always $x_{0}=60$, and the highest attainable age is $\omega=100$. The annuities have deferral periods ranging from $T=6$ to $T=20$ years, which means that they are respectively associated with years-of-birth from 1958 to 1972 (i.e., current ages from 59 to 45). As the largest year-ofbirth covered by the data sample is $t_{b}-x_{a}=2011-50=1961$, some of the annuity liabilities are subject to cohort effect uncertainty but some are not.

The hedge portfolio consists of $m=1 \mathrm{q}$-forward. It is assumed that three q-forwards with the same reference age $\left(x_{1}=65\right)$ but different times-to-maturity $\left(T_{1}=11,16,21\right)$ are available in the market. These combinations of $x_{1}$ and $T_{1}$ imply that the q-forwards are associated with years-of-birth 1957, 1962 and 1967, respectively.

The hedge is established at the end of year $t_{b}=2011$ (i.e., at the same time when the annuities are sold). We consider a hedging horizon of $\tau=1$ year for the following two reasons. First, the results for $\tau=1$ are the most informative, as a dynamic hedge is simply a repeated execution of static hedges with $\tau=1$. Second, when $\tau=1$, some of the analytical expressions in Section 4 can be simplified, thereby allowing us to more easily link the empirical results to the theoretical work. When valuing the annuity liabilities and the q-forwards, a continuously compounded risk-adjusted discount rate of $r=0.01$ per annum is assumed.

The relationships between the calculated hedge effectiveness and the annuity's period of deferral under different circumstances are displayed in Figure 5. Separate panels are used to distinguish the results based on different q-forward specifications. Each panel shows two sets of results that are obtained from the same collection of sample paths: (1) the results that are based on the proposed calibration method with cohort effect uncertainty taken into consideration (the solid line 


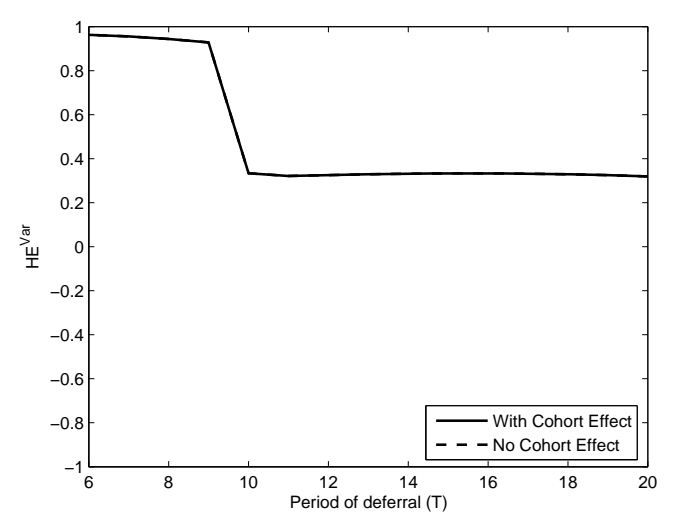

(a) $x_{1}=65, T_{1}=11$

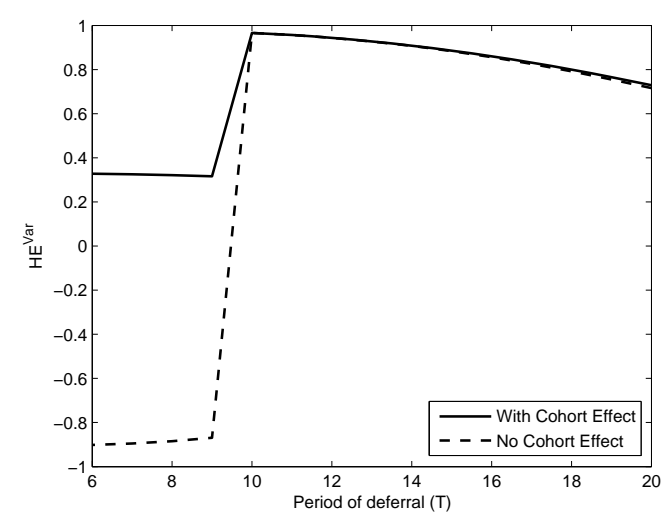

(b) $x_{1}=65, T_{1}=16$

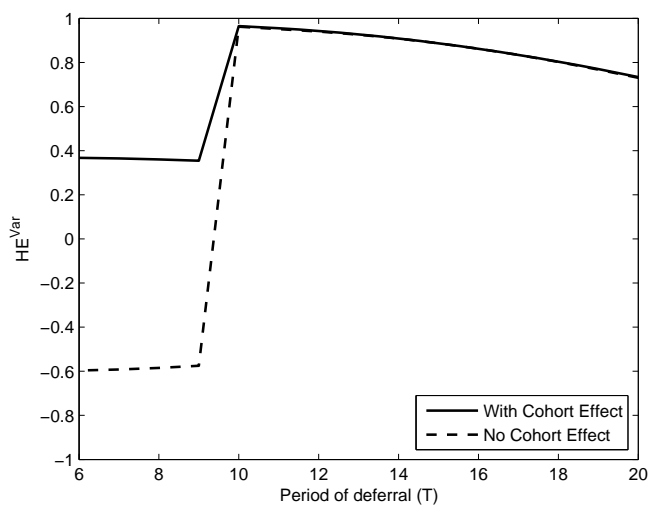

(c) $x_{1}=65, T_{1}=21$

Figure 5: The relationship between $\mathrm{HE}^{\mathrm{Var}}$ and the period of deferral $T$ for different $\mathrm{q}$-forward choices, estimated using the baseline parameters.

labeled "with cohort effect"); (2) the results that are based on the proposed calibration method but cohort effect uncertainty is ignored (the dashed line labeled "no cohort effect"). A comparison between these two sets of results is made in Section 6.2.

A striking observation is that there is an (asymmetric) spike at $T=10$. More interestingly, the spike in panel (a) points downwards (i.e., a sharp reduction when $T$ increases from 9 to 10) while the spikes in panels (b) and (c) point upwards (i.e., a sharp increase when $T$ increases from 9 to 10). We can explain the spikes in the patterns of $\mathrm{HE}^{\mathrm{Var}}$ by noting that the analytical approximation of the optimized $\mathrm{HE}^{\mathrm{Var}}$ is given by

$$
1-\frac{k_{3}-\frac{k_{2}^{2}}{k_{1}}}{k_{3}}=\frac{k_{2}^{2}}{k_{1} k_{3}},
$$

Let $\vec{\eta}_{t}^{p}=\left(\eta_{t}^{(1)}, \eta_{t}^{(2)}, \eta_{t}^{(3)}\right)^{\prime}$ be the vector of the time- $t$ innovations in the period effect processes. 
When $\tau=1$,

$$
\begin{aligned}
k_{1} & =\left(\frac{\partial H_{t_{b}}\left(1, t_{b}\right)}{\partial \vec{\eta}_{t_{b}+1}}\right)^{\prime} Q^{*}\left(\frac{\partial H_{t_{b}}\left(1, t_{b}\right)}{\partial \vec{\eta}_{t_{b}+1}}\right) \\
& =\left(\frac{\partial H_{t_{b}}\left(1, t_{b}\right)}{\partial \vec{\eta}_{t_{b}+1}^{p}}\right)^{\prime} Q\left(\frac{\partial H_{t_{b}}\left(1, t_{b}\right)}{\partial \vec{\eta}_{t_{b}+1}^{p}}\right)+\left(\frac{\partial H_{t_{b}}\left(1, t_{b}\right)}{\partial \eta_{t_{b}-x_{a}+1}^{(4)}}\right)^{2}\left(\sigma^{(4)}\right)^{2}
\end{aligned}
$$

which is positively related to $\partial H_{t_{b}}\left(1, t_{b}\right) / \partial \eta_{t_{b}-x_{a}+1}^{(4)}$,

$$
\begin{aligned}
k_{3} & =\left(\frac{\partial L_{t_{b}}}{\partial \vec{\eta}_{t_{b}+1}}\right)^{\prime} Q^{*}\left(\frac{\partial L_{t_{b}}}{\partial \vec{\eta}_{t_{b}+1}}\right) \\
& =\left(\frac{\partial L_{t_{b}}}{\partial \vec{\eta}_{t_{b}+1}^{p}}\right)^{\prime} Q\left(\frac{\partial L_{t_{b}}}{\partial \vec{\eta}_{t_{b}+1}^{p}}\right)+\left(\frac{\partial L_{t_{b}}}{\partial \eta_{t_{b}-x_{a}+1}^{(4)}}\right)^{2}\left(\sigma^{(4)}\right)^{2}
\end{aligned}
$$

which is positively related to $\partial L_{t_{b}} / \partial \eta_{t_{b}-x_{a}+1}^{(4)}$, and

$$
\begin{aligned}
k_{2} & =\left(\frac{\partial L_{t_{b}}}{\partial \vec{\eta}_{t_{b}+1}}\right)^{\prime} Q^{*}\left(\frac{\partial H_{t_{b}}\left(1, t_{b}\right)}{\partial \vec{\eta}_{t_{b}}+1}\right) \\
& =\left(\frac{\partial L_{t_{b}}}{\partial \vec{\eta}_{t_{b}+1}^{p}}\right)^{\prime} Q\left(\frac{\partial H_{t_{b}}\left(1, t_{b}\right)}{\partial \vec{\eta}_{t_{b}+1}^{p}}\right)+\left(\frac{\partial H_{t_{b}}\left(1, t_{b}\right)}{\partial \eta_{t_{b}-x_{a}+1}^{(4)}}\right)\left(\frac{\partial L_{t_{b}}}{\partial \eta_{t_{b}-x_{a}+1}^{(4)}}\right)\left(\sigma^{(4)}\right)^{2}
\end{aligned}
$$

which is positively related to the product of $\partial H_{t_{b}}\left(1, t_{b}\right) / \partial \eta_{t_{b}-x_{a}+1}^{(4)}$ and $\partial L_{t_{b}} / \partial \eta_{t_{b}-x_{a}+1}^{(4)} \cdot{ }^{16}$ Furthermore,

$$
\frac{\partial H_{t_{b}}\left(1, t_{b}\right)}{\partial \eta_{t_{b}-x_{a}+1}^{(4)}}=-X\left(T_{1}-x_{1}+x_{a}-1\right) e^{-r T_{1}}\left(1-q_{x_{1}, t_{b}+T_{1}}\right) q_{x_{1}, t_{b}+T_{1}}
$$

is proportional to $X\left(T_{1}-x_{1}+x_{a}-1\right)$, and

$$
\frac{\partial L_{t}}{\partial \eta_{t_{b}-x_{a}+1}^{(4)}}=-X\left(x_{a}-x_{0}+T\right) \sum_{u=1}^{\omega-x_{0}} e^{-r(T+u)}\left(\prod_{s=1}^{T+u}\left(1-q_{x_{0}-T+s-1, t_{b}+s}\right)\right)\left(\sum_{s=t-t_{b}+1}^{T+u} q_{x_{0}-T+s-1, t_{b}+s}\right)
$$

is proportional to $X\left(x_{a}-x_{0}+T\right)$. The following conclusions can then be drawn.

- $x_{1}=65$ and $T_{1}=11($ panel (a))

We have $X\left(x_{a}-x_{0}+T\right)=X(50-60+9)=X(-1)=0$ at $T=9$ and $X\left(x_{a}-x_{0}+T\right)=$ $X(50-60+10)=X(0)=1$ at $T=10$. As a result, when $T$ increases from 9 to $10, k_{3}$ (which is positively related to $\left.X\left(x_{a}-x_{0}+T\right)\right)$ increases sharply. On the other hand, $k_{1}$ remains unchanged because it is unrelated to the annuity liability (and thus the annuity's deferral

\footnotetext{
${ }^{16}$ A larger value of $\partial H_{t_{b}}\left(1, t_{b}\right) / \partial \eta_{t_{b}-x_{a}+1}^{(4)}$ means that $H_{t_{b}}\left(1, t_{b}\right)$ is more sensitive to $\eta_{t_{b}-x_{a}+1}^{(4)}$. As such, $H_{t_{b}}\left(1, t_{b}\right)$ is subject to more cohort effect uncertainty, and therefore $k_{1}$ - which represents the (approximate) variance $H_{t_{b}}\left(1, t_{b}\right)$ - would increase if $\partial H_{t_{b}}\left(1, t_{b}\right) / \partial \eta_{t_{b}-x_{a}+1}^{(4)}$ is larger. Similar arguments can be used to explain the other two relationships.
} 
period $T)$. Also, $k_{2}$ remains unchanged, because it is related to $X\left(x_{a}-x_{0}+T\right)$ through the product of $\partial L_{t_{b}} / \partial \eta_{t_{b}-x_{a}+1}^{(4)}$ and $\partial H_{t_{b}}\left(1, t_{b}\right) / \partial \eta_{t_{b}-x_{a}+1}^{(4)}$, but $\partial H_{t_{b}}\left(1, t_{b}\right) / \partial \eta_{t_{b}-x_{a}+1}^{(4)}=0$ when $x_{1}=65$ and $T_{1}=11$ (because the cohort to which the q-forward is linked is already realized at $\left.t_{b}=2011\right)$. Overall, as $T$ increases from 9 to 10, the denominator of expression (6.1) increases sharply but the numerator remains unchanged, rendering a downward spike in the optimized hedge effectiveness.

- $x_{1}=65$ and $T_{1}=16($ panel (b))

As in the previous scenario, $k_{1}$ remains unchanged while $k_{3}$ increases sharply as $T$ increases from 9 to 10. Nevertheless, for this q-forward specification, we have $X\left(T_{1}-x_{1}+x_{a}-1\right)=$ $X(16-65+50-1)=X(0)=1($ instead of 0$)$. Therefore, when $T$ increases from 9 to 10 , $X\left(x_{a}-x_{0}+T\right)$ changes from 0 to 1 and consequently $k_{2}$ (which is positively related to the product of $X\left(x_{a}-x_{0}+T\right)$ and $\left.X\left(T_{1}-x_{1}+x_{a}-1\right)\right)$ increase sharply. Overall, as $T$ increases from 9 to 10, both the numerator and denominator of expression (6.1) increase significantly, resulting in an optimized hedge effectiveness that is much closer to 1.

- $x_{1}=65$ and $T_{1}=21$ (panel (c))

The situation here is exactly the same as that when $x_{1}=65$ and $T_{1}=16$, except that $X\left(T_{1}-x_{1}+x_{a}-1\right)$ becomes $X(21-65+50-1)=X(5)$. Given the baseline estimates of $\theta_{1}^{(4)}$ and $\phi_{1}^{(4)}, X(5)$ is 0.9146 , which is rather close to 1 . Therefore, we still observe an upward spike in the optimized hedge effectiveness. We remark here that for other estimates of $\theta_{1}^{(4)}$ and $\phi_{1}^{(4)}$, the value of $X(5)$ may be much smaller than 1 (closer to 0 ) and in this case the increase in $k_{2}$ would be mild compared to that in $k_{3}$, which would result in a downward spike instead. This phenomenon is demonstrated in the next two sub-sections where alternative ARMA parameters for the cohort effect process are considered.

\subsection{The Benefit of Incorporating Cohort Effects}

We now evaluate the benefit of incorporating cohort effect by comparing the solid and dotted lines in Figure 5. We observe that hedge effectiveness can be negative if cohort effect uncertainty is not incorporated into the hedging strategy. This outcome is not overly surprising, as there is no guarantee that variance can be reduced if the actual (simulation) model is different from the model on which the hedging strategy is based (see, e.g., Li et al, 2016).

The gap between the solid and dashed lines reflects the benefit of incorporating cohort effect uncertainty into the hedging strategy. When the q-forward with $x_{1}=65$ and $T_{1}=11$ is used (panel (a)), the lines coincide. This outcome is because the q-forward, which is linked to a cohort (year-of-birth 1957) that is already realized in the data sample, is not subject to any cohort effect uncertainty. The q-forward is thus unable to mitigate any cohort effect uncertainty involved in the annuity liability no matter how the hedge is calibrated. The other two q-forwards are related 
to unrealized cohorts (years-of-birth 1962 and 1967), so a gap between the lines exists when either one of them is used. The benefit of incorporating cohort effect uncertainty seems more remarkable when the q-forward with $x_{1}=65$ and $T_{1}=16$ (panel (b)) is used.

To explain why the benefit of incorporating cohort effect uncertainty depends on the q-forward specification, let us revisit the optimal hedging strategies derived earlier. Recall that for a static hedge with $m=1$ q-forward, the optimized notional amount when cohort effect uncertainty is ignored is

$$
\hat{\mathcal{N}}_{1}\left(t_{b}\right)=\frac{k_{2}\left(1-\frac{c_{2}}{k_{2}}\right)}{k_{1}\left(1-\frac{c_{1}}{k_{1}}\right)},
$$

whereas the optimized notional amount when cohort effect uncertainty is taken into account is

$$
\hat{N}_{1}\left(t_{b}\right)=\frac{k_{2}}{k_{1}}
$$

These two notional amounts (and hence the corresponding hedge effectiveness values) are close to each other if

$$
\frac{c_{1}}{k_{1}}=\frac{\left(\frac{\partial H_{t_{b}}\left(1, t_{b}\right)}{\partial \eta_{t_{b}-x_{a}+1}^{(4)}}\right)^{2}\left(\sigma^{(4)}\right)^{2}}{\left(\frac{\partial H_{t_{b}}\left(1, t_{b}\right)}{\partial \vec{\eta}_{t_{b}+1}}\right)^{\prime} Q^{*}\left(\frac{\partial H_{t_{b}}\left(1, t_{b}\right)}{\partial \vec{\eta}_{t_{b}+1}}\right)},
$$

the proportion of the variance of $H_{t_{b}}\left(1, t_{b}\right)$ that is contributed from cohort effect uncertainty, and

$$
\frac{c_{2}}{k_{2}}=\frac{\left(\frac{\partial H_{t_{b}}\left(1, t_{b}\right)}{\partial \eta_{t_{b}-x_{a}+1}^{(4)}}\right)\left(\frac{\partial L_{t_{b}}}{\partial \eta_{t_{b}-x_{a}+1}^{(4)}}\right)\left(\sigma^{(4)}\right)^{2}}{\left(\frac{\partial L_{t_{b}}}{\partial \vec{\eta}_{t_{b}+1}}\right)^{\prime} Q^{*}\left(\frac{\partial H_{t_{b}}\left(1, t_{b}\right)}{\partial \vec{\eta}_{t_{b}+1}}\right)}
$$

the proportion of the covariance between $H_{t_{b}}\left(1, t_{b}\right)$ and $L_{t_{b}}$ that is contributed from cohort effect uncertainty, are close to each other.

Table 2 reports the values of $c_{1} / k_{1}$ and $c_{2} / k_{2}$ for different combinations of the q-forward's maturity $\left(T_{1}\right)$ and the liability's deferral period $(T)$ when the q-forward's reference age $x_{1}$ is fixed to 65 . For $T_{1} \leq 15$, both ratios are zero because in this case $H_{t_{b}}\left(1, t_{b}\right)$ is not related to any unrealized cohort. This fact again explains why the two lines in panel (a) coincide.

For $T=5$ (more generally $T<10$ ), $c_{2} / k_{2}=0$ for all values of $T_{1}$ because in this case $L_{t}$ is not related to any unrealized cohort. Also, for $T=5$, the value of $c_{1} / k_{1}$ is higher when $T_{1}=16$ than when $T_{1}=21$, explaining why the gap between the solid and dashed lines (for $T<10$ ) is wider in panel (b) than in panel (c).

For $T \geq 10\left(\right.$ and $\left.T_{1} \geq 16\right), c_{1} / k_{1}$ and $c_{2} / k_{2}$ are very close. The proximity between $c_{1} / k_{1}$ and 
Table 2: The values of $c_{1} / k_{1}$ and $c_{2} / k_{2}$ for different combinations of the q-forward's maturity $\left(T_{1}\right)$ and the liability's deferral period $(T)$, when the q-forward's reference age $x_{1}$ is fixed to 65 . The values are calculated using the baseline parameters.

\begin{tabular}{|c|c|c|c|c|c|c|c|}
\hline$T_{1}$ & $\leq 15$ & 16 & 17 & 18 & 19 & 20 & 21 \\
\hline & \multicolumn{7}{|c|}{$\bar{T}=20$} \\
\hline$c_{1} / k_{1}$ & 0 & 0.6595 & 0.6665 & 0.6547 & 0.6427 & 0.6306 & 0.6183 \\
\hline \multirow[t]{2}{*}{$c_{2} / k_{2}$} & 0 & 0.6138 & 0.6175 & 0.6112 & 0.6050 & 0.5987 & 0.5924 \\
\hline & \multicolumn{7}{|c|}{$T=15$} \\
\hline$c_{1} / k_{1}$ & 0 & 0.6595 & 0.6665 & 0.6547 & 0.6427 & 0.6306 & 0.6183 \\
\hline \multirow[t]{2}{*}{$c_{2} / k_{2}$} & 0 & 0.6422 & 0.6458 & 0.6398 & 0.6337 & 0.6276 & 0.6214 \\
\hline & \multicolumn{7}{|c|}{$T=10$} \\
\hline$c_{1} / k_{1}$ & 0 & 0.6595 & 0.6665 & 0.6547 & 0.6427 & 0.6306 & 0.6183 \\
\hline \multirow[t]{2}{*}{$c_{2} / k_{2}$} & 0 & 0.6572 & 0.6607 & 0.6548 & 0.6488 & 0.6428 & 0.6368 \\
\hline & \multicolumn{7}{|c|}{$T=5$} \\
\hline$c_{1} / k_{1}$ & 0 & 0.6595 & 0.6665 & 0.6547 & 0.6427 & 0.6306 & 0.6183 \\
\hline$c_{2} / k_{2}$ & 0 & 0 & 0 & 0 & 0 & 0 & 0 \\
\hline
\end{tabular}

$c_{2} / k_{2}$ explains why for $T \geq 10$ the gaps between the solid and dashed lines in panels (b) and (c) are almost unobservable. Note that this outcome is not a general phenomenon. To illustrate, we consider an alternative set of ARMA parameters $\left(\phi_{1}^{(4)}=0.6, \theta_{1}^{(4)}=-0.4\right)$ for the cohort effect process. The hedge effectiveness and the ratios of $c_{1} / k_{1}$ and $c_{2} / k_{2}$ when the alternative ARMA parameters are used are presented in Figure 6 and Table 3, respectively. Given the alternative ARMA parameters, the differences between $c_{1} / k_{1}$ and $c_{2} / k_{2}$ for $T>10$ and $T_{1}=16$ (i.e., when the annuity liability is associated with an unrealized cohort which is different from the cohort to which the q-forward is linked) become significant. As such, when the q-forward with $T_{1}=16$ is used, the benefit of incorporating cohort effect uncertainty becomes very apparent.

The results shown in Figures 5 and 6 highlight an important fact: even if the annuity liability is not linked to any unrealized cohort, incorporating cohort effect uncertainty into the calibration process may still result in a more effective hedge. We can explain this fact by considering again the expressions for $\hat{\mathcal{N}}_{1}\left(t_{b}\right)$ and $\hat{N}_{1}\left(t_{b}\right)$. When the annuity liability is not linked to any unrealized cohort, $c_{2}$ equals zero. However, $c_{1}$ may still be positive (and large in magnitude) as it is not related to the annuity liability. Therefore, $\hat{\mathcal{N}}_{1}\left(t_{b}\right)$ may still be significantly different from $\hat{N}_{1}\left(t_{b}\right)$, which means the incorporation of cohort effect uncertainty still matters.

One final interesting observation from Figure 6 is that when $x_{1}=65$ and $T_{1}=16$ (i.e., when the q-forward is linked to year-of-birth 1962), the gap between the solid and dashed lines is significant for the entire range of $T$ except $T=10$ (i.e., when the annuity liability is also linked to year-of-birth 1962). To explain why, we consider the following two facts.

First, when the annuity liability and the q-forward are associated with the same year-of-birth, we have $t_{b}+T_{1}-x_{1}=t_{b}-\left(x_{0}-T\right)+1$, which implies $T_{1}-x_{1}+x_{a}-1=x_{a}-x_{0}+T=C_{t_{b}, 1}$. 


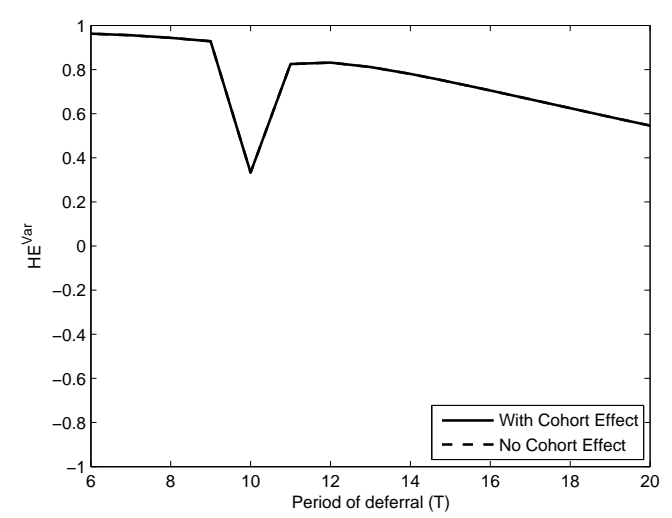

(a) $x_{1}=65, T_{1}=11$

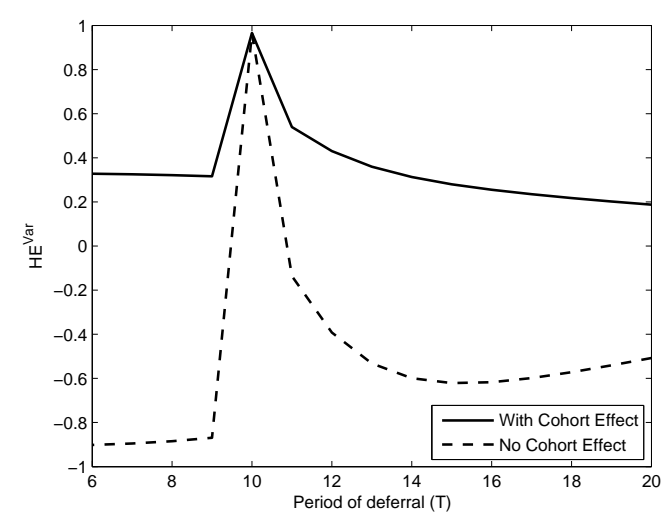

(b) $x_{1}=65, T_{1}=16$

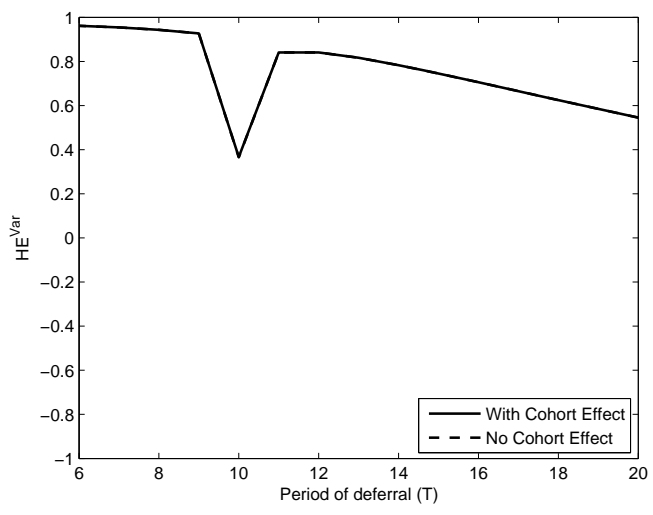

(c) $x_{1}=65, T_{1}=21$

Figure 6: The relationship between $\mathrm{HE}^{\mathrm{Var}}$ and the period of deferral $T$ for different $\mathrm{q}$-forward choices, estimated using the alternative ARMA parameters $\left(\phi_{1}^{(4)}=0.6, \theta_{1}^{(4)}=-0.4\right)$.

Also, for the given values of $t_{a}, t_{b}, x_{a}, T_{1}$ and $x_{1}$, we have $C_{t_{b}, 1}=0$. It follows that

$$
X\left(T_{1}-x_{1}+x_{a}-1\right)=X\left(C_{t_{b}, 1}\right)=X(0)=1 .
$$

Then, by substituting $k=1$ and $X\left(T_{1}-x_{1}+x_{a}-1\right)=1$ into equation (4.11) and $t=t_{b}$ and $X\left(C_{t_{b}, 1}\right)$ into equation (4.8), we obtain the following:

$$
\frac{\partial H_{t_{b}}\left(1, t_{b}\right)}{\partial \eta_{t_{b}+1}^{(1)}}=\frac{\partial H_{t_{b}}\left(1, t_{b}\right)}{\partial \eta_{t_{b}-x_{a}+1}^{(4)}} \quad \text { and } \quad \frac{\partial L_{t_{b}}}{\partial \eta_{t_{b}+1}^{(1)}}=\frac{\partial L_{t_{b}}}{\partial \eta_{t_{b}-x_{a}+1}^{(4)}}
$$

Second, it is found empirically that

$$
k_{1} \approx\left(\frac{\partial H_{t_{b}}\left(1, t_{b}\right)}{\partial \eta_{t_{b}+1}^{(1)}}\right)^{2} Q^{*}(1,1)+\left(\frac{\partial H_{t_{b}}\left(1, t_{b}\right)}{\partial \eta_{t_{b}-x_{a}+1}^{(4)}}\right)^{2}\left(\sigma^{(4)}\right)^{2}
$$


Table 3: The values of $c_{1} / k_{1}$ and $c_{2} / k_{2}$ for different combinations of the q-forward's maturity $\left(T_{1}\right)$ and the liability's deferral period $(T)$, when the q-forward's reference age $x_{1}$ is fixed to 65 .

The values are calculated using alternative ARMA parameters $\left(\phi_{1}^{(4)}=0.6, \theta_{1}^{(4)}=-0.4\right)$.

\begin{tabular}{c|ccccccc}
\hline \hline$T_{1}$ & $\leq 15$ & 16 & 17 & 18 & 19 & 20 & 21 \\
\hline \hline & \multicolumn{7}{|c}{$T=20$} \\
\hline$c_{1} / k_{1}$ & 0 & 0.6595 & 0.0719 & 0.0271 & 0.0099 & 0.0036 & 0.0013 \\
$c_{2} / k_{2}$ & 0 & 0.0040 & 0.0008 & 0.0005 & 0.0003 & 0.0002 & 0.0001 \\
\hline \hline & \multicolumn{7}{|c}{$T=15$} \\
\hline$c_{1} / k_{1}$ & 0 & 0.6595 & 0.0719 & 0.0271 & 0.0099 & 0.0036 & 0.0013 \\
$c_{2} / k_{2}$ & 0 & 0.0485 & 0.0101 & 0.0061 & 0.0037 & 0.0022 & 0.0013 \\
\hline \hline & \multicolumn{7}{|c}{$T=10$} \\
\hline$c_{1} / k_{1}$ & 0 & 0.6595 & 0.0719 & 0.0271 & 0.0099 & 0.0036 & 0.0013 \\
$c_{2} / k_{2}$ & 0 & 0.6573 & 0.2773 & 0.1871 & 0.1214 & 0.0765 & 0.0474 \\
\hline \hline & \multicolumn{7}{c}{$T=5$} \\
\hline$c_{1} / k_{1}$ & 0 & 0.6595 & 0.0719 & 0.0271 & 0.0099 & 0.0036 & 0.0013 \\
$c_{2} / k_{2}$ & 0 & 0 & 0 & 0 & 0 & 0 & 0 \\
\hline \hline
\end{tabular}

and

$$
k_{2} \approx\left(\frac{\partial H_{t_{b}}\left(1, t_{b}\right)}{\partial \eta_{t_{b}+1}^{(1)}}\right)\left(\frac{\partial L_{t_{b}}}{\partial \eta_{t_{b}+1}^{(1)}}\right) Q^{*}(1,1)+\left(\frac{\partial H_{t_{b}}\left(1, t_{b}\right)}{\partial \eta_{t_{b}-x_{a}+1}^{(4)}}\right)\left(\frac{\partial L_{t_{b}}}{\partial \eta_{t_{b}-x_{a}+1}^{(4)}}\right)\left(\sigma^{(4)}\right)^{2}
$$

which is due in part to the fact that $Q^{*}(1,1)$ (the variance of $\left.\eta_{t}^{(1)}\right)$ and $Q^{*}(4,4)\left(\left(\sigma^{(4)}\right)^{2}\right.$, the variance of $\left.\eta_{t-x}^{(4)}\right)$ are significantly larger than the other elements in $Q^{*}$ (see Table 1 ).

Using these two facts and equations (6.7) and (6.8), we can deduce that if the annuity liability and the q-forward are linked to the same cohort, then $c_{1} / k_{1}$ would be close to $c_{2} / k_{2}$ and consequently $\hat{\mathcal{N}}_{1}\left(t_{b}\right)$ would be close to $\hat{N}_{1}\left(t_{b}\right) .{ }^{17}$ Therefore, in this case, the sub-optimality due to the ignorance of cohort effect uncertainty is minimal.

\subsection{The Impact of the Persistency in the Cohort Effects}

The baseline results reveal that the benefit from incorporating cohort effect uncertainty depends critically on $X(s)$, which in turn depends on parameters $\theta_{1}^{(4)}$ and $\phi_{1}^{(4)}$. As $X(s)$ represents the coefficient of the lag-s innovation in infinite MA form of the cohort effect process (equation (4.2)), it may be regarded as a measure of the persistency in the cohort effects. In particular, if $X(s)$ decays rapidly as $s$ increases from zero, then an innovation in a certain year-of-birth would have only a small impact on the cohort effect a few years-of-birth ahead; and the opposite is true if the decay in $X(s)$ is slow.

\footnotetext{
${ }^{17}$ If the approximations in equations $(6.9)$ and $(6.10)$ are exact, then $c_{1} / k_{1}=c_{2} / k_{2}=\left(\sigma^{(4)}\right)^{2} /\left(Q *(1,1)+\left(\sigma^{(4)}\right)^{2}\right)$ The benefit of incorporating cohort effect uncertainly would therefore be exactly zero.
} 


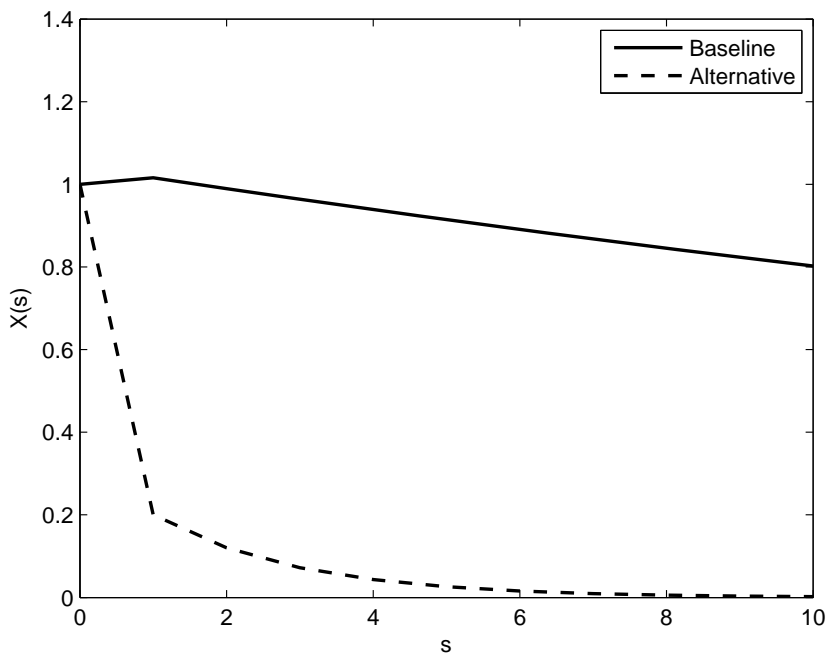

Figure 7: The patterns of $X(s)$ implied by the baseline ARMA parameters $\left(\theta_{1}^{(4)}=0.0417\right.$, $\left.\phi_{1}^{(4)}=0.9741\right)$ and the alternative ARMA parameters $\left(\theta_{1}^{(4)}=-0.4, \phi_{1}^{(4)}=0.6\right)$. Note: $X(s)=0$ for $s<0$ regardless of the parameter choice.

In this sub-section, we study how and why the hedging results may change with the persistency of cohort effects. To achieve this goal, we compare the hedging results derived from the baseline parameters and the alternative parameters used in Section 6.2. The former implies a high cohort effect persistency as $\theta_{1}^{(4)}+\phi_{1}^{(4)}=0.0417+0.9741=1.0158$, while the latter implies a low cohort effect persistency as $\theta_{1}^{(4)}+\phi_{1}^{(4)}=0.6+(-0.4)=0.2$. The degrees of persistency implied by the two sets of ARMA parameters are demonstrated in Figure 7 . Except the assumptions about $\theta_{1}^{(4)}$ and $\phi_{1}^{(4)}$, all assumptions remain unchanged.

The hedging results derived using the baseline and alternative ARMA parameters are shown in Figure 8. The similarities and differences between the two sets of results can be explained by considering the following properties, most of which are discussed in Section 6.1:

1. the optimized hedge effectiveness is $k_{2}^{2} /\left(k_{1} k_{3}\right)$ (equation (6.1));

2. the optimized hedge effectiveness $k_{2}^{2} /\left(k_{1} k_{3}\right)$ is approximately the squared correlation between $L_{t_{b}}$ and $H_{t_{b}}\left(1, t_{b}\right)$;

3. the ARMA parameters affect $k_{1}$ through $\partial H_{t_{b}}\left(1, t_{b}\right) / \partial \eta_{t_{b}-x_{a}+1}^{(4)}$ (equation (6.2));

4. the ARMA parameters affect $k_{2}$ through the product of $\partial H_{t_{b}}\left(1, t_{b}\right) / \partial \eta_{t_{b}-x_{a}+1}^{(4)}$ and $\partial L_{t_{b}} / \partial \eta_{t_{b}-x_{a}+1}^{(4)}$ (equation (6.3));

5. the ARMA parameters affect $k_{3}$ through $\partial L_{t_{b}} / \partial \eta_{t_{b}-x_{a}+1}^{(4)}$ (equation (6.4));

6. $\partial H_{t_{b}}\left(1, t_{b}\right) / \partial \eta_{t_{b}-x_{a}+1}^{(4)}$ is proportional to $X\left(T_{1}-x_{1}+x_{a}-1\right)$ (equation (6.5)); 
7. $\partial L_{t_{b}} / \partial \eta_{t_{b}-x_{a}+1}^{(4)}$ is proportional to $X\left(x_{a}-x_{0}+T\right)$ (equation $\left.(6.6)\right)$

For panel (a), the q-forward with $x_{1}=65$ and $T_{1}=11$ is linked to a realized cohort (year-ofbirth 1957). Given that the q-forward is linked to a realized cohort, $\partial H_{t_{b}}\left(1, t_{b}\right) / \partial \eta_{t_{b}-x_{a}+1}^{(4)}$ must be 0 and hence the values of $k_{1}$ and $k_{2}$ remain unchanged no matter what ARMA parameters are used. However, the behaviour of $k_{3}$ depends on the value of $T$.

- For $T<10$, the liability is also linked to a realized cohort, which means $\partial L_{t_{b}} / \partial \eta_{t_{b}-x_{a}+1}^{(4)}$ must also be 0 and thus $k_{3}$ is also invariant with the ARMA parameters. As a result, both parameter sets lead to the same optimized hedge effectiveness.

- For $T=10$, the value of $X\left(x_{a}-x_{0}+T\right)=X(50-60+10)=X(0)$ is 1 regardless of what ARMA parameters are used. Consequently, $k_{3}$ and the optimized hedge effectiveness are still invariant with ARMA parameters.

- For $T>10$, the value of $X\left(x_{a}-x_{0}+T\right)=X(50-60+T)=X(T-10)$ depends on the ARMA parameters and so does $\partial L_{t_{b}} / \partial \eta_{t_{b}-x_{a}+1}^{(4)}$. When the alternative parameters are used, the values of $X(T-10)$ for $T>10$ are much smaller (see Figure 7 ), leading to a much lower $k_{3}$ and hence a significantly higher optimized hedge effectiveness $k_{2}^{2} /\left(k_{1} k_{3}\right)$.

For panel (b), the q-forward with $x_{1}=65$ and $T_{1}=16$ is linked to an unrealized cohort (yearof-birth 1962). Given this q-forward specification, $X\left(T_{1}-x_{1}+x_{a}-1\right)=X(16-65+50-1)=X(0)$ must be 1 no matter what ARMA parameters are used. Hence, the value of $k_{1}$ is invariant with the ARMA parameters. Let us study the following scenarios.

- For $T<10$, the liability is linked to a realized cohort, which means $\partial L_{t_{b}} / \partial \eta_{t_{b}-x_{a}+1}^{(4)}$ must be 0 and hence $k_{2}$ and $k_{3}$ are also invariant with the ARMA parameters. Overall, both parameter sets lead to the same optimized hedge effectiveness.

- For $T=10$, the value of $X\left(x_{a}-x_{0}+T\right)=X(50-60+10)=X(0)$ is 1 regardless of what ARMA parameters are used. Hence, $k_{2}$ and $k_{3}$ are still invariant with ARMA parameters, and so is the optimized hedge effectiveness.

- For $T>10, L_{t_{b}}$ and $H_{t_{b}}\left(t_{b}, 1\right)$ are linked to different cohorts, both of which are unrealized. In this case, other things equal, the correlation between $L_{t_{b}}$ and $H_{t_{b}}\left(t_{b}, 1\right)$ is higher if the cohort effects are more persistent. ${ }^{18}$ As the optimized hedge effectiveness $k_{2}^{2} /\left(k_{1} k_{3}\right)$ is approximately the squared correlation between $L_{t_{b}}$ and $H_{t_{b}}\left(1, t_{b}\right)$, the higher the cohort effect persistency is, the higher the optimized hedge effectiveness is.

\footnotetext{
${ }^{18}$ Consider two unrealized cohort effects: $\gamma_{t_{b}-x_{a}+u}^{(4)}$ and $\gamma_{t_{b}-x_{a}+v}^{(4)}$, where $v>u>1$. If persistency is high, then the innovation $\eta_{t_{b}-x_{a}+1}^{(4)}$ for year-of-birth $t_{b}-x_{a}+1$ would affect $\gamma_{t_{b}-x_{a}+u}^{(4)}$ and $\gamma_{t_{b}-x_{a}+v}^{(4)}$ similarly, which in turn means that $\gamma_{t_{b}-x_{a}+v}^{(4)}$ is more strongly correlated with $\gamma_{t_{b}-x_{a}+u}^{(4)}$. If persistency is low, then the impact of $\eta_{t_{b}-x_{a}+1}^{(4)}$ on $\gamma_{t_{b}-x_{a}+v}^{(4)}$ would be much smaller than that on $\gamma_{t_{b}-x_{a}+u}^{(4)}$, which in turn means that $\gamma_{t_{b}-x_{a}+v}^{(4)}$ is more weakly correlated with $\gamma_{t_{b}-x_{a}+u}^{(4)}$.
} 
For panel (c), the q-forward with $x_{1}=65$ and $T_{1}=21$ is also linked to an unrealized cohort (year-of-birth 1967). We observe the following two features that are not found in the previous two panels.

- For $T<10$, the two parameter sets leads to very different optimized hedge effectiveness. As in panel (b), $k_{2}$ and $k_{3}$ for $T<10$ are invariant with the ARMA parameters. However, for this q-forward specification, the values of $k_{1}$-which is positively related to $X\left(T_{1}-x_{1}+\right.$ $\left.x_{a}-1\right)=X(21-65+50-1)=X(5)$ - under the two sets of ARMA parameters are very different. In particular, while $X(5)$ is 0.9146 under the baseline ARMA parameters, it is only 0.0259 under the alternative ARMA parameters. Therefore, when the alternative ARMA parameters are used, $k_{1}$ is substantially smaller and the optimized hedge effectiveness $k_{2}^{2} /\left(k_{1} k_{3}\right)$ is much higher.

- Under the alternative ARMA parameters, the spike at $T=10$ points downwards instead of upwards. As discussed in Section 6.1, when $T$ increases from 9 to 10, $k_{1}$ remains unchanged, $k_{3}$ increases abruptly, and the increase in $k_{2}$ is positively related to the value of $X\left(T_{1}-\right.$ $\left.x_{1}+x_{a}-1\right)=X(21-65+50-1)=X(5)$. Under the alternative parameter set, $X(5)$ is rather close to zero (0.0259). As such, when $T$ increases from 9 to 10 , the increase in $k_{2}$ is much milder compared to that in $k_{3}$, leading to a sudden drop in the optimized hedge effectiveness.

\subsection{The Effect of $\tau$}

So far, we have assumed that the hedging horizon is $\tau=1$ year. In this sub-section we sensitivity test $\tau$, while keeping all other assumptions unchanged.

Figure 9 shows the relationships between the optimized hedge effectiveness $\mathrm{HE}^{\mathrm{Var}}$ and the hedging horizon $\tau$, derived using the baseline parameters and the alternative ARMA parameters

$\left(\phi_{1}^{(4)}=0.6, \theta_{1}^{(4)}=-0.4\right)$. The results are based on a deferral period of $T=20$ years and a q-forward with a reference age of $x_{1}=65$ and a time-to-maturity of $T_{1}=16$ years.

For $\tau \leq T_{1}=16$, the relationship between the optimized $\mathrm{HE}^{\mathrm{Var}}$ and $\tau$ takes no definite shape. This can be understood by noting that the optimized $\mathrm{HE}^{\mathrm{Var}}$ is $k_{2}^{2} /\left(k_{1} k_{3}\right)$ and by revisiting the expressions for $k_{1}, k_{2}$ and $k_{3}$ (equations (4.15) to (4.17)). For $\tau \leq T_{1}, k_{1}, k_{2}$ and $k_{3}$ all increase monotonically with $\tau$, as every term in the summands in equations (4.15) to (4.17) is non-negative. Hence, the optimized $\mathrm{HE}^{\mathrm{Var}}$ increases with $\tau$ if $k_{2}^{2}$ increases at a faster rate than $k_{1} k_{3}$, and vice versa.

However, for $\tau>T_{1}$, the optimized $\mathrm{HE}^{\mathrm{Var}}$ must be strictly decreasing with $\tau$. This is because $k_{1}$ and $k_{2}$ no longer vary with $\tau$, as $\partial H_{t_{b}}\left(1, t_{b}\right) / \partial \vec{\eta}_{t_{b}+s}=0$ for $s>T_{1}$ (the value of the q-forward is unrelated to any innovation vector beyond the q-forward's maturity), but $k_{3}$ remains increasing with $\tau$. In effect, the optimized hedge effectiveness $k_{2}^{2} /\left(k_{1} k_{3}\right)$ must reduce with $\tau$. 


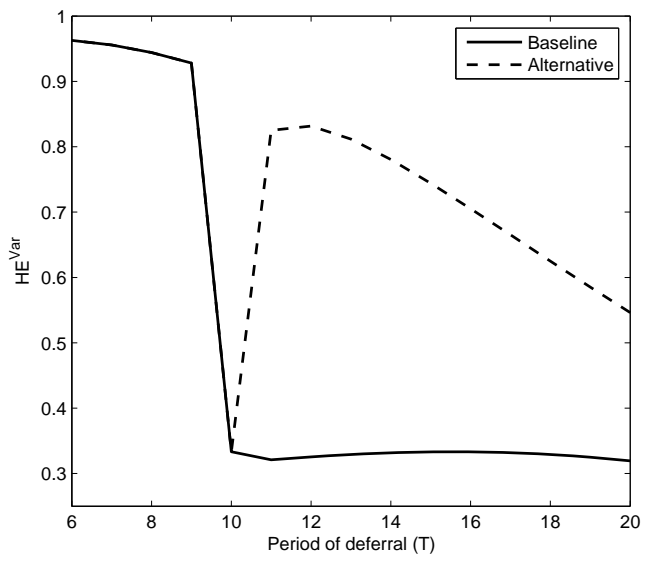

(a) $x_{1}=65, T_{1}=11$

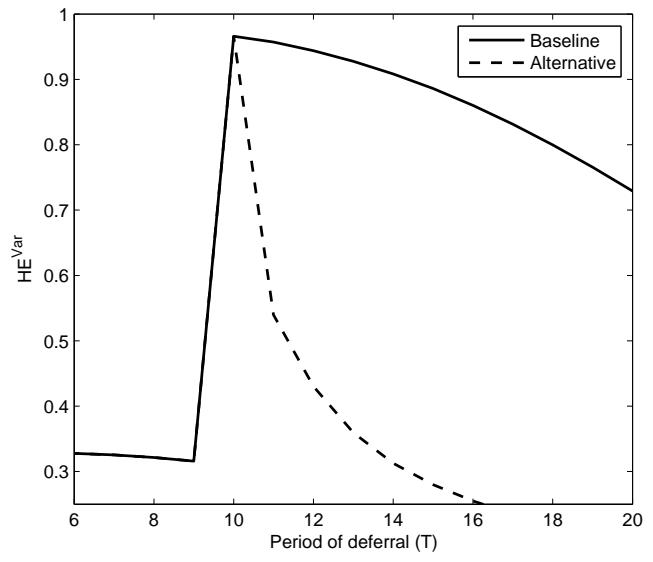

(b) $x_{1}=65, T_{1}=16$

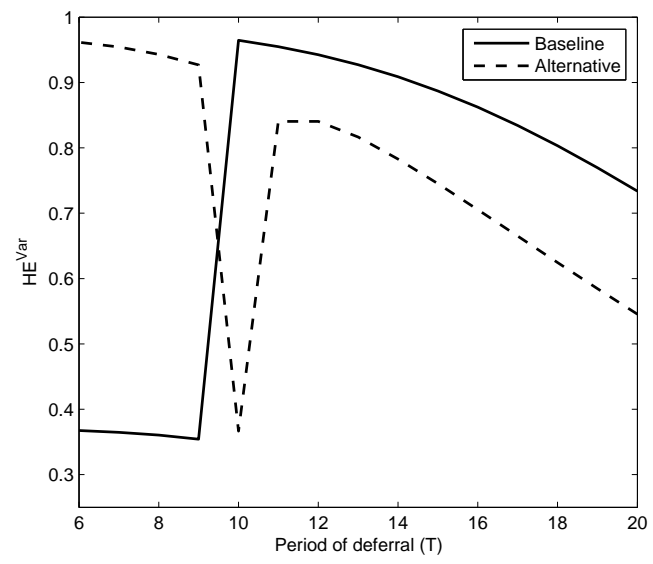

(c) $x_{1}=65, T_{1}=21$

Figure 8: The calculated values of $\mathrm{HE}^{\mathrm{Var}}$ under the baseline ARMA parameters $\left(\theta_{1}^{(4)}=0.0417\right.$, $\left.\phi_{1}^{(4)}=0.9741\right)$ and the alternative ARMA parameters $\left(\theta_{1}^{(4)}=-0.4, \phi_{1}^{(4)}=0.6\right)$.

\section{Conclusion}

Cohort effect uncertainty often plays a role in an index-based longevity hedge, because either the liability being hedged, the hedging instrument, or both are associated with cohorts that are not yet realized. However, it is largely overlooked in the existing methods for calibrating index-based longevity hedges. When cohort effect uncertainty is present but is not taken into account in the hedging strategy, the resulting longevity hedge may perform sub-optimally.

In this paper, we have contributed a hedge calibration method that fully incorporates cohort effect uncertainty. With the proposed method, one can find the notional amounts that minimizes the variance of the $\tau$-year ahead values of the hedged position. The optimized notional amounts can be expressed in terms of $Q^{*}$ (the covariance matrix of the innovation vector) and various partial 


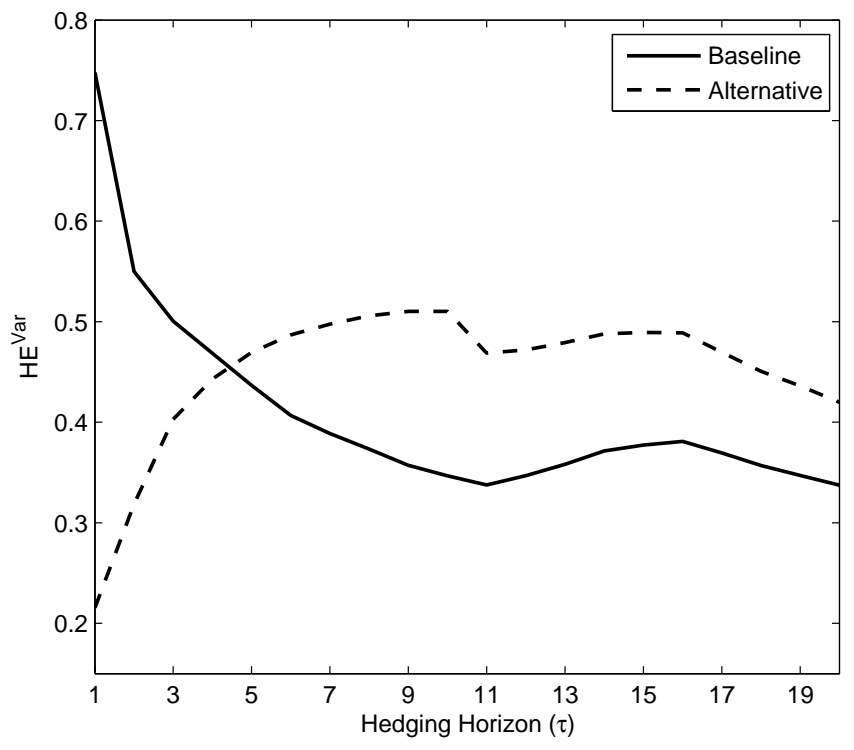

Figure 9: The calculated values of $\mathrm{HE}^{\mathrm{Var}}$ for different hedging horizons, on the basis of the baseline parameters and the alternative ARMA parameters $\left(\phi_{1}^{(4)}=0.6, \theta_{1}^{(4)}=-0.4\right)$. It is assumed that $T=20, x_{1}=65$ and $T_{1}=16$.

derivatives that can be analytically computed. By setting $\tau=1$ and executing the calibration method every year, an annually-adjusted dynamic longevity hedge can be created easily.

The proposed methods have been illustrated using historical mortality data from English and Welsh male population, for which cohort effects are found to be significant. Through an illustrative longevity hedge with one q-forward, we have examined the benefit of incorporating cohort effects under different circumstances. A number of lessons can be learnt from the empirical work.

First, even if the liability being hedged is not linked to any unrealized cohort, incorporating cohort effect uncertainty into the calibration process may still yield a more effective hedge. This outcome is because the optimal notional amount depends on the sensitivity of the q-forward's value to the underlying cohort effect. As long as the q-forward is linked to an unrealized cohort, ignoring this component would result in a sub-optimal notional amount.

Second, the benefit of incorporating cohort effect uncertainty is small when the liability being hedged and the q-forward are linked to the same cohort, because under this condition $c_{1} / k_{1}$ and $c_{2} / k_{2}$ are close to each other. This finding is complementary to a conclusion obtained by Cairns et al. (2014): a longevity hedge (without incorporating cohort effects) tends to be less effective when there is a mismatch between the cohorts to which the hedging instrument and the liability being hedged are linked. When the problem of mismatched cohorts is inevitable, the hedger should be mindful of the less than ideal hedge effectiveness and factor cohort effect uncertainty into his/her hedging strategy.

Third and last, the hedging results depend heavily on $X(s)$, which measures the persistency of 
cohort effects. Two examples are summarized here. (1) When the q-forward is free of cohort effect uncertainty but the annuity liability is not, then the hedge effectiveness would be higher when cohort effect persistency is lighter. (2) If the annuity liability is free of cohort effect uncertainty but the q-forward is linked to an unrealized cohort that is a few years ahead of the most recently realized cohort, then the hedge would be more effective when cohort effects are less persistent.

The key limitations of this paper merit further discussions. When $t-x$ is close to $t_{b}-x_{a}$ or $t_{a}-x_{b}$ (the latest or earliest years-of-birth covered by the data sample), the estimates of $\gamma_{t-x}^{(4)}$ in Model M7 are based on only a few data points and therefore subject to significant parameter uncertainty (the uncertainty involved in parameter estimation). In turn, there are reliability issues surrounding the parameters in the cohort effect process. Partly because of this, we sensitivity tested the ARMA parameters in the cohort effect process (Section 6.3) by examining how the hedging results may change under (very) different parameter combinations.

Our modeling and hedging framework depends on a sharp distinction between realized cohorts (those born in or before year $t_{b}-x_{a}$ ) and unrealized cohorts (those born after $t_{b}-x_{a}$ ). This sharp distinction may be seen as undesirable, because, as mentioned in the previous paragraph, not all realized cohorts are equally known. Further, if we alter the sample age range and/or the sample period to which the model is fitted, the distinction between realized and unrealized cohorts change and so do the hedging results. For instance, if we extend the sample age range from 50-89 to 49-89, then one more cohort (that born in 1962) would be classified as a realized cohort and consequently the position of the spike in $\mathrm{HE}^{\mathrm{Var}}$ (Figures 5 and 8) would be shifted from $T=10$ to $T=11$.

The abrupt change in $\mathrm{HE}^{\mathrm{Var}}$ as $T$ increases from 9 to 10 also represents a limitation. This limitation is inherited from the way in which cohort effects are captured by Model M7 (and most if not all stochastic mortality models that incorporate cohort effects): through a time-series process, stochastic uncertainty (uncertainty captured by the random innovations) is attached to cohorts not covered by the data sample; but all cohorts covered by the data sample are given absolutely no stochastic uncertainty, regardless of the fact that we have little information concerning the youngest and oldest ones. Removing the oldest and youngest cohorts in the data sample (Cairns et al., 2014) does not mitigate the problem, as the sharp cutoff between unrealized cohorts (which are subject to stochastic uncertainty) and realized cohorts (which are assumed to be totally free of stochastic uncertainty) still remains. We believe that this limitation may be overcome in two different directions:

1. Incorporate parameter uncertainty into the modeling work, and then develop a hedging strategy that mitigates parameter uncertainty on top of stochastic uncertainty.

2. Develop a new modeling approach in which some stochastic uncertainty is attached to cohorts that are only partially observed.

Both approaches do not seem straightforward, and demand significant further research. 
To focus on cohort effect uncertainty, we do not consider population basis risk in this paper. However, with the aid of a multi-population mortality model, we can easily extend the proposed calibration method to incorporate the difference between the populations involved. For example, if we assume the augmented common factor model ( $\mathrm{Li}$ and Lee, 2005), then we can derive the hedging strategy by first formulating $L_{t}$ and $H_{t}(j, t)$ as functions of the innovations from the random walk that captures the common mortality trend and the autoregressive processes that characterize the population-specific mortality trends. We also ignore sampling risk, which is likely to be very critical if a higher value of $\omega$ is used. To gauge the impact of sampling risk, one may use a discrete distribution such as Poisson to model death counts conditioned on the realized underlying mortality rate (see, e.g., Cairn et al., 2014). We leave these extensions to future research.

\section{Acknowledgments}

This work is supported by research grants from the Global Risk Institute, the Natural Sciences and Engineering Research Council of Canada (Discovery Grant RGPIN-356050-2013) and the Society of Actuaries Center of Actuarial Excellence Program.

\section{References}

Blake, D., Cairns, A., Coughlan, G., Dowd, K. and MacMinn, R. (2013). The New Life Market. Journal of Risk and Insurance. 80, 501-557.

Blake, D., MacMinn, R., Li, J.S.-H. and Hardy, M. (2014). Longevity Risk and Capital Markets: The 2012-2013 Update. North American Actuarial Journal, 18, 501-557.

Box, G.E.P. and Jenkins, G.M. (1976). Time Series Analysis: Forecasting and Control. 2nd edition. San Francisco: Holden-Day.

Cairns, A.J.G. (2011). Modelling and Management of Longevity Risk: Approximations to Survival Functions and Dynamic Hedging. Insurance: Mathematics and Economics, 49, 438-453.

Cairns, A.J.G. (2013). Robust Hedging of Longevity Risk. Journal of Risk and Insurance, 80, 621-648.

Cairns, A.J.G., Blake, D. and Dowd, K., (2006a). A Two-Factor Model for Stochastic Mortality with Parameter Uncertainty: Theory and Calibration. Journal of Risk and Insurance, 73, 687-718.

Cairns, A.J.G., Blake, D., Dowd, K. and Coughlan, G.D. (2014). Longevity Hedge Effectiveness: A Decomposition. Quantitative Finance, 14, 217-235.

Cairns, A.J.G., Blake, D., Dowd, K., Coughlan, G.D., Epstein, D., and Khalaf-Allah, M. (2011). Mortality Density Forecasts: An Analysis of Six Stochastic Mortality Models. Insurance: Mathematics and Economics, 48, 355-367. 
Cairns, A.J.G., Blake, D., Dowd, K., Coughlan, G.D., Epstein, D., Ong, A., and Balevich, I. (2009). A Quantitative Comparison of Stochastic Mortality Models Using Data from England and Wales and the United States. North American Actuarial Journal, 13, 1-35.

Cairns, A.J.G., Blake, D., Dowd, K., and Kessler, A.R. (2016). Phantoms Never Die: Living with Unreliable Population Data. Journal of the Royal Statistical Society: Series A (Statistics in Society). DOI: $10.1111 /$ rssa.12159.

Cairns, A., Blake, D., Dowd, K. and MacMinn, R., (2006b). Longevity Bonds: Financial Engineering, Valuation, and Hedging. Journal of Risk and Insurance, 73, 647-672.

Continuous Mortality Investigation Bureau (2002). An Interim Basis for Adjusting the "92" Series Mortality Projections for Cohort Effects. CMI Working Paper 1. London: Institute of Actuaries and Faculty of Actuaries.

Coughlan, G., Blake, D., MacMinn, R., Cairns, A.J.G. and Dowd, K. (2013). Longevity Risk and Hedging Solutions. In Handbook of Insurance (pp. 997-1035). Springer: New York.

Coughlan, G.D., Khalaf-Allah, M., Ye, Y., Kumar, S., Cairns, A.J.G., Blake, D. and Dowd, K. (2011). Longevity Hedging 101: A Framework for Longevity Basis Risk Analysis and Hedge Effectiveness. North American Actuarial Journal, 15, 150-176.

Chuang, S.-L. and Brockett, P.L. (2014). Modeling and Pricing Longevity Derivatives using Stochastic Mortality Rates and the Esscher Transform. North American Actuarial Journal, 18, 22-37.

Dahl, M. (2004). Stochastic Mortality in Life Insurance: Market Reserves and Mortality-Linked Insurance Contracts. Insurance: Mathematics and Economics, 35, 113-136.

Dahl, M., Melchior, M. and Møller, T. (2008). On Systematic Mortality Risk and Risk Minimization with Mortality Swaps. Scandinavian Actuarial Journal, 108, 114-146.

Dahl, M. and Møller, T. (2006). Valuation and Hedging of Life Insurance Liabilities with Systematic Mortality Risk. Insurance: Mathematics and Economics, 39, 193-217.

Denuit, M. and Dhaene, J. (2007). Comonotonic Bounds on the Survival Probability in the Lee-Carter Model for Mortality Projection. Computational and Applied Mathematics, 203, 169-176.

Denuit, M., Haberman, S. and Renshaw, A.E. (2010). Comonotonic Approximations to Quantiles of Life Annuity Conditional Expected Present Values: Extensions to General ARIMA Models and Comparison with the Bootstrap. ASTIN Bulletin, 40, 331-349.

Dowd, K., Cairns, A.J.G., Blake, D., Coughlan, G.D., Epstein, D., and Khalaf-Allah, M. (2010a). Evaluating the Goodness of Fit of Stochastic Mortality Models. Insurance: Mathematics and Economics, 47, 255-265.

Dowd, K., Cairns, A.J.G., Blake, D., Coughlan, G.D., Epstein, D., and Khalaf-Allah, M. (2010b). Backtesting Stochastic Mortality Models: An Ex-Post Evaluation of Multi-Period-Ahead Density Forecasts. North American Actuarial Journal, 14, 281-298.

Gong, G. and Webb, A. (2010). Evaluating the Advanced Life Deferred Annuity - An Annuity People Might Actually Buy. Insurance: Mathematics and Economics, 46, 210-221. 
Haberman, S., Kaishev, V., Millossovich, P, Villegas, A., Baxter, S. Gaches, A., Gunnlaugsson, S. and Sison, M. (2014). Longevity Basis Risk: A Methodology for Assessing Basis Risk. Research investigation and report by Cass Business School and Hymans Robertson LLP for the Institute and Faculty of Actuaries and the Life and Longevity Markets Association. Available at http://www.actuaries.org.uk/research-and-resources/documents/sessional-research-event-longevitybasis-risk-methodology

Holmes, E.C. (2004). Enhanced: 1918 and All That. Science, 303, 1787-1788.

Horneff, W., Maurer, R. and Rogalla, R. (2010). Dynamic Portfolio Choice with Deferred Annuities. Journal of Banking and Finance, 34, 2652-2664.

Human Mortality Database. University of California, Berkeley (USA), and Max Planck Institute of Demographic Research (Germany). Available at www.mortality.org or www.humanmortality.de (data downloaded on 1 April 2015).

Hunt, A. and Blake, D. (2015). Forward Mortality Rates in Discrete Time I: Calibration and Securities Pricing. Pensions Institute Discussion Paper PI-1511.

Hunt, A. and Blake, D. (2016). Forward Mortality Rates in Discrete Time II: Longevity Risk and Hedging Strategies. Pensions Institute Discussion Paper PI-1602.

Li, J.S.-H., Chan, W.-S., and Zhou, R. (2016), Semicoherent Multipopulation Mortality Modeling: The Impact on Longevity Risk Securitization. Journal Risk and Insurance. doi:10.1111/jori.12135

Li, J.S.-H. and Hardy, M.R. (2011). Measuring Basis Risk in Longevity Hedges. North American Actuarial Journal, 15, 177-200.

Li, J.S.-H., Hardy, M. R., and Tan, K.S. (2009). Uncertainty in Mortality Forecasting: An Extension to the Classical Lee-Carter Approach. ASTIN Bulletin, 39, 137-164.

Li, J.S.-H. and Luo, A. (2012). Key q-duration: A Framework for Hedging Longevity Risk. ASTIN Bulletin, 42, 413-452.

Li, J.S.-H., Ng, A.C.Y. and Chan, W.S. (2011). On the Calibration of Mortality Forward Curves. Journal of Futures Markets, 31, 941-970.

Li, N. and Lee, R. (2005). Coherent Mortality Forecasts for a Group of Populations: An Extension of the Lee-Carter Method. Demography. 42, 575-594.

Liu, Y. and Li, J.S.-H. (2014). The Locally-Linear Cairns-Blake-Dowd Model: A Note on Delta-Nuga Hedging of Longevity Risk. Paper presented at the 10th International Longevity Risk and Capital Markets Solutions Conference, Santiago, Chile.

Luciano, E., Regis, L. and Vigna, E. (2012). Delta-Gamma Hedging of Mortality and Interest Rate Risk. Insurance: Mathematics and Economics, 50, 402-412.

Milevsky, M. (2005). Real Longevity Insurance with a Deductible: Introduction to Advanced-Life Delayed Annuities. North American Actuarial Journal, 9, 109-122. 
Ngai, A. and Sherris, M. (2011). Longevity Risk Management for Life and Variable Annuities: The Effectiveness of Static Hedging Using Longevity Bonds and Derivatives. Insurance Mathematics and Economics, 49, 100-114.

Richards, S.J. (2008). Detecting Year-of-Birth Mortality Patterns with Limited Data. Journal of the Royal Statistical Society: Series A (Statistics in Society), 171, 279-298.

Villegas, A.M., Millossovich, P. and Kaishev, V.K. (2016). StMoMo: An R Package for Stochastic Mortality Modelling. R package version 0.3.1. URL http://CRAN.R-project.org/package=StMoMo

Zhou, K.Q. and Li, J.S.-H. (2014). Dynamic Longevity Hedging in the Presence of Population Basis Risk: A Feasibility Analysis from Technical and Economic Perspectives. Paper presented at the 10th International Longevity Risk and Capital Markets Solutions Conference, Santiago, Chile.

\section{Appendix A:}

Deriving the Partial Derivatives with Respect to $\eta_{t+k}^{(1)}, \eta_{t+k}^{(2)}$ and $\eta_{t+k}^{(3)}$

The partial derivatives of $L_{t}$ with respect to $\eta_{t+k}^{(1)}, \eta_{t+k}^{(2)}$ and $\eta_{t+k}^{(3)}$ for $k=1, \ldots, \omega-x_{0}+T+\left(t_{b}-t\right)$ (presented in equation (4.7)) are obtained as follows:

$$
\begin{aligned}
\frac{\partial L_{t}}{\partial \eta_{t+k}^{(i)}} & =\sum_{u=1}^{\omega-x_{0}} e^{-r(T+u)}\left(\prod_{s=1}^{t-t_{b}}\left(1-q_{x_{0}-T+s-1, t_{b}+s}\right)\right) \frac{\partial\left(\prod_{s=t-t_{b}+1}^{T+u}\left(1-q_{x_{0}-T+s-1, t_{b}+s}\right)\right)}{\partial \eta_{t+k}^{(i)}} \\
& =\sum_{u=1}^{\omega-x_{0}} e^{-r(T+u)}\left(\prod_{s=1}^{t-t_{b}+k-1}\left(1-q_{x_{0}-T+s-1, t_{b}+s}\right)\right) \frac{\partial\left(\begin{array}{c}
T+u \\
\prod_{s=t-t_{b}+k}^{T}\left(1-q_{x_{0}-T+s-1, t_{b}+s}\right)
\end{array}\right)}{\partial \eta_{t+k}^{(i)}},
\end{aligned}
$$

where

$$
\begin{aligned}
& \frac{\partial\left(\prod_{s=t-t_{b}+k}^{T+u}\left(1-q_{x_{0}-T+s-1, t_{b}+s}\right)\right)}{\partial \eta_{t+k}^{(i)}}=\frac{\partial\left(\prod_{s=t-t_{b}+k}^{T+u}\left(1-q_{x_{0}}-T+s-1, t_{b}+s\right)\right.}{\partial \eta_{t+k}^{(i)}} \\
& \left.=\sum_{v=t-t_{b}+k}^{T+u}\left(\frac{\partial\left(\prod_{s=t-t_{b}+k}^{T+u}\left(1-q_{x_{0}-T+s-1, t_{b}+s}\right)\right.}{\partial q_{x_{0}}-T+v-1, t_{b}+v}\right) \cdot \frac{\partial q_{x_{0}-T+v-1, t_{b}+v}}{\partial \kappa_{t+k}^{(i)}} \cdot \frac{\partial \kappa_{t+k}^{(i)}}{\partial \eta_{t+k}^{(i)}}\right) \\
& =-\sum_{v=t-t_{b}+k}^{T+u}\left(\left(\prod_{\substack{s=t-t_{b}+k \\
s \neq v}}^{T+u}\left(1-q_{x_{0}-T+s-1, t_{b}+s}\right)\right) \cdot \frac{\partial q_{x_{0}-T+v-1, t_{b}+v}}{\partial \kappa_{t+k}^{(i)}} \cdot \frac{\partial \kappa_{t+k}^{(i)}}{\partial \eta_{t+k}^{(i)}}\right), \\
& \frac{\partial q_{x_{0}-T+v-1, t_{b}+v}}{\partial \kappa_{t+k}^{(i)}}= \begin{cases}\left(q_{x_{0}-T+v-1, t_{b}+v}\right)\left(1-q_{x_{0}-T+v-1, t_{b}+v}\right), & \text { for } i=1 \\
\left(x_{0}-T+v-1-\bar{x}\right)\left(q_{x_{0}-T+v-1, t_{b}+v}\right)\left(1-q_{x_{0}-T+v-1, t_{b}+v}\right), & \text { for } i=2 \\
\left(\left(x_{0}-T+v-1-\bar{x}\right)^{2}-\hat{\sigma}_{x}^{2}\right)\left(q_{x_{0}-T+v-1, t_{b}+v}\right)\left(1-q_{x_{0}-T+v-1, t_{b}+v}\right), & \text { for } i=3\end{cases}
\end{aligned}
$$


Similarly, the partial derivatives of $H_{j}(j, t)$ with respect to $\eta_{t+k}^{(1)}, \eta_{t+k}^{(2)}$ and $\eta_{t+k}^{(3)}$ for $k=1, \ldots, T_{j}$ (presented in equation (4.10)) are derived as follows:

$$
\begin{aligned}
\frac{\partial H_{j}(j, t)}{\partial \eta_{t+k}^{(i)}} & =-e^{-r T_{j}} \frac{\partial q_{x_{j}, t+T_{j}}}{\partial \eta_{t+k}^{(i)}} \\
& =-e^{-r T_{j}} \frac{\partial q_{x_{j}, t+T_{j}}}{\partial \kappa_{t+k}^{(i)}} \cdot \frac{\partial \kappa_{t+k}^{(i)}}{\partial \eta_{t+k}^{(i)}}
\end{aligned}
$$

where

$$
\frac{\partial q_{x_{j}, t+T_{j}}}{\partial \kappa_{t+k}^{(i)}}=\left\{\begin{array}{ll}
\left(q_{x_{j}, t+T_{j}}\right)\left(1-q_{x_{j}, t+T_{j}}\right), & \text { for } i=1 \\
\left(x_{j}-\bar{x}\right)\left(q_{x_{j}, t+T_{j}}\right)\left(1-q_{x_{j}, t+T_{j}}\right), & \text { for } i=2 \\
\left(\left(x_{j}-\bar{x}\right)^{2}-\hat{\sigma}_{x}^{2}\right)\left(q_{x_{j}, t+T_{j}}\right)\left(1-q_{x_{j}, t+T_{j}}\right), & \text { for } i=3
\end{array},\right.
$$

and $\frac{\partial \kappa_{t+k}^{(i)}}{\partial \eta_{t+k}^{(i)}}=1$ for $i=1,2,3$

\section{Appendix B:}

\section{Deriving the Partial Derivatives with Respect to $\eta_{t-x_{a}+k}^{(4)}$ and $\eta_{t+T_{j}-x_{j}}^{(4)}$}

The partial derivative of $L_{t}$ with respect to $\eta_{t-x_{a}+k}^{(4)}$ for $k=1, \ldots, t_{b}-x_{0}+T+1-t+x_{a}$ (presented in equation (4.8)) is evaluated as follows:

$$
\frac{\partial L_{t}}{\partial \eta_{t-x_{a}+k}^{(4)}}=\sum_{u=1}^{\omega-x_{0}} e^{-r(T+u)}\left(\prod_{s=1}^{t-t_{b}}\left(1-q_{x_{0}-T+s-1, t_{b}+s}\right)\right) \frac{\partial\left(\prod_{s=t-t_{b}+1}^{T+u}\left(1-q_{x_{0}-T+s-1, t_{b}+s}\right)\right)}{\partial \eta_{t-x_{a}+k}^{(4)}},
$$

where

$$
\begin{aligned}
& \frac{\partial\left(\prod_{s=t-t_{b}+1}^{T+u}\left(1-q_{x_{0}-T+s-1, t_{b}+s}\right)\right)}{\partial \eta_{t-x_{a}+k}^{(4)}} \\
= & \sum_{v=t-t_{b}+1}^{T+u}\left(\frac{\partial\left(\prod_{s=t-t_{b}+1}^{T+u}\left(1-q_{x_{0}-T+s-1, t_{b}+s}\right)\right)}{\partial q_{x_{0}-T+v-1, t_{b}+v}} \cdot \frac{\partial q_{x_{0}-T+v-1, t_{b}+v}}{\partial \gamma_{t_{b}-\left(x_{0}-T\right)+1}^{(4)}} \cdot \frac{\partial \gamma_{t_{b}-\left(x_{0}-T\right)+1}^{(4)}}{\partial \eta_{t-x_{a}+k}^{(4)}}\right) \\
= & -\sum_{v=t-t_{b}+1}^{T+u}\left(\left(\prod_{\substack{s=t-t_{b}+1 \\
s \neq v}}^{T+u}\left(1-q_{x_{0}-T+s-1, t_{b}+s}\right)\right) \cdot \frac{\partial q_{x_{0}-T+v-1, t_{b}+v}}{\partial \gamma_{t_{b}-\left(x_{0}-T\right)+1}^{(4)}} \cdot \frac{\partial \gamma_{t_{b}-\left(x_{0}-T\right)+1}^{(4)}}{\partial \eta_{t-x_{a}+k}^{(4)}}\right),
\end{aligned}
$$

and

$$
\frac{\partial q_{x_{0}-T+v-1, t_{b}+v}}{\partial \gamma_{t_{b}-\left(x_{0}-T\right)+1}^{(4)}}=q_{x_{0}-T+v-1, t_{b}+v} \cdot\left(1-q_{x_{0}-T+v-1, t_{b}+v}\right) .
$$


The value of $\partial \gamma_{t_{b}-\left(x_{0}-T\right)+1}^{(4)} / \partial \eta_{t-x_{a}+k}^{(4)}$ depends on $C_{t, k}=\left(t_{b}-\left(x_{0}-T\right)+1\right)-\left(t-x_{a}+k\right)$, the difference between the following two quantities:

1. the year-of-birth associated with the annuity liability $\left(t_{b}-\left(x_{0}-T\right)+1\right)$;

2. the year-of-birth associated with the innovation under consideration $\left(t-x_{a}+k\right)$;

In particular, we have

$$
\frac{\partial \gamma_{t_{b}-\left(x_{0}-T\right)+1}^{(4)}}{\partial \eta_{t-x_{a}+k}^{(4)}}=X\left(C_{t, k}\right)
$$

where $X(\cdot)$ is specified in equation (4.9), which is obtained by induction.

Similarly, the partial derivative of $H_{t}(j, t)$ with respect to $\eta_{t-x_{a}+k}^{(4)}$ for $k=1, \ldots, T_{j}-x_{j}+x_{a}$ (presented in equation (4.11)) is derived as follows:

$$
\begin{aligned}
\frac{\partial H_{t}(j, t)}{\partial \eta_{t-x_{a}+k}^{(4)}} & =-e^{-r T_{j}} \frac{\partial q_{x_{j}, t+T_{j}}}{\partial \eta_{t-x_{a}+k}^{(4)}} \\
& =-e^{-r T_{j}} \frac{\partial q_{x_{j}, t+T_{j}}}{\partial \gamma_{t+T_{j}-x_{j}}^{(4)}} \cdot \frac{\partial \gamma_{t+T_{j}-x_{j}}^{(4)}}{\partial \eta_{t-x_{a}+k}^{(4)}}
\end{aligned}
$$

where

$$
\frac{\partial q_{x_{j}, t+T_{j}}}{\partial \gamma_{t+T_{j}-x_{j}}^{(4)}}=q_{x_{j}, t+T_{j}} \cdot\left(1-q_{x_{j}, t+T_{j}}\right) .
$$

The value of $\partial \gamma_{t+T_{j}-x_{j}}^{(4)} / \partial \eta_{t-x_{a}+k}^{(4)}$ depends on $T_{j}-x_{j}+x_{a}-k$, which represents the difference between the following two quantities:

1. the year-of-birth associated with the hedging instrument $\left(t+T_{j}-x_{j}\right)$;

2. the year-of-birth associated with the innovation under consideration $\left(t-x_{a}+k\right)$.

Specifically, we have

$$
\frac{\partial \gamma_{t+T_{j}-x_{j}}^{(4)}}{\partial \eta_{t-x_{a}+k}^{(4)}}=X\left(T_{j}-x_{j}+x_{a}-k\right),
$$

where $X(\cdot)$ is specified in equation (4.9). 